TITLE:

\title{
The Random Assignment Problem with Submodular Constraints on Goods
}

\author{
AUTHOR(S): \\ Fujishige, Satoru; Sano, Yoshio; Zhan, Ping
}

CITATION:

Fujishige, Satoru ...[et al]. The Random Assignment Problem with Submodular

Constraints on Goods. ACM Transactions on Economics and Computation 2018, 6(1): 3 .

ISSUE DATE:

2018-01-22

URL:

http://hdl.handle.net/2433/228999

\section{RIGHT:}

(c) ACM, 2018. This is the author's version of the work. It is posted here by permission of ACM for your personal use. Not for redistribution. The definitive version was published in PUBLICATION, [Volume 6 Issue 1, January 2018, Article No. 3] http://doi.acm.org/10.1145/3175496; この論文は出版社版でありません。引用の際には出版社版をご確認ご利用くだ さい。; This is not the published version. Please cite only the published version. 


\title{
The Random Assignment Problem with Submodular Constraints on Goods
}

\author{
SATORU FUJISHIGE* ${ }^{*}$ Kyoto University, Japan \\ YOSHIO SANO, University of Tsukuba, Japan \\ PING ZHAN, Edogawa University, Japan
}

Problems of allocating indivisible goods to agents in an efficient and fair manner without money have long been investigated in the literature. The random assignment problem is one of them, where we are given a fixed feasible (available) set of indivisible goods and a profile of ordinal preferences over the goods, one for each agent. Then, using lotteries, we determine an assignment of goods to agents in a randomized way. A seminal paper of Bogomolnaia and Moulin (2001) shows a probabilistic serial (PS) mechanism to give an ordinally efficient and envy-free solution to the assignment problem.

In this paper we consider an extension of the random assignment problem to submodular constraints on goods. We show that the approach of the PS mechanism by Bogomolnaia and Moulin is powerful enough to solve the random assignment problem with submodular (matroidal and polymatroidal) constraints. Under the agents' ordinal preferences over goods we show the following.

(1) The obtained PS solution for the problem with unit demands and matroidal constraints is ordinally efficient, envy-free, and weakly strategy-proof with respect to the associated stochastic dominance relation.

(2) For the multi-unit demand and polymatroidal constraint problem the PS solution is ordinally efficient and envy-free but is not strategy-proof in general. However, we show that under a mild condition (that is likely to be satisfied in practice) the PS solution is a weak Nash equilibrium.

CCS Concepts: • Theory of computation $\rightarrow$ Algorithmic game theory and mechanism design; Discrete optimization;

Additional Key Words and Phrases: Random assignment, probabilistic serial mechanism, ordinal preference, matchings, polymatroids, independent flows, submodular optimization, weak Nash equilibrium

ACM Reference Format:

Satoru FUJISHIGE, Yoshio SANO, and Ping ZHAN. 2017. The Random Assignment Problem with Submodular Constraints on Goods. 1, 1, Article 1 (October 2017), 27 pages.

https://doi.org/10.1145/nnnnnnn.nnnnnnn

\section{INTRODUCTION}

Problems of allocating indivisible goods to agents in a fair and efficient manner without money have long been investigated in the literature (see, e.g., $[1-6,17-22,26,28,31])$. Suppose that we are given a fixed feasible (available) set of indivisible goods and a profile of ordinal preferences over the goods, one for each agent. Then, using lotteries, we determine an assignment of goods to agents in a randomized way. A seminal paper of Bogomolnaia

*The corresponding author

S. Fujishige's work is supported by JSPS KAKENHI Grant Number JP25280004 and Y. Sano's work by JSPS KAKENHI Grant Numbers JP15K20885, JP16H03118.

ACM acknowledges that this contribution was authored or co-authored by an employee, contractor, or affiliate of the United States government. As such, the United States government retains a nonexclusive, royalty-free right to publish or reproduce this article, or to allow others to do so, for government purposes only.

(c) 2017 Association for Computing Machinery.

XXXX-XXXX/2017/10-ART1 \$15.00

https://doi.org/10.1145/nnnnnnn.nnnnnnn

, Vol. 1, No. 1, Article 1. Publication date: October 2017 
and Moulin [5] shows a probabilistic serial (PS) mechanism to give an ordinally efficient and envy-free solution to the assignment problem.

In this paper we consider an extension of the random assignment problem to submodular constraints on goods in two cases:

(1) Agents have unit demands and the family of feasible sets of goods forms a family of bases of a matroid. (The original problem in [5] is concerned with a matroid having only one base.)

(2) Agents have multi-unit demands and the set of feasible integral vectors of goods forms an integral polymatroid. (A polymatroid having only one base is treated in $[6,18,21]$.)

We show that the approach of the PS mechanism by Bogomolnaia and Moulin [5] is powerful enough to solve the random assignment problem with submodular (matroidal and polymatroidal) constraints. Under the agents' ordinal preferences over goods we prove the following.

(1) The obtained PS solution for the problem with unit demands and matroidal constraints is ordinally efficient, envy-free, and weakly strategy-proof with respect to the partial order defined by the stochastic dominance relation introduced by Bogomolnaia and Moulin [5].

(2) For the multi-unit demand and polymatroidal constraint problem the PS solution is ordinally efficient and envy-free but is not weakly strategy-proof in general. However, under a mild condition (that is likely to be satisfied in practice) the PS solution is a weak Nash equilibrium.

The well-known Birkhoff-von Neumann theorem on bi-stochastic matrices shows that every bi-stochastic matrix is expressed as a convex combination of permutation matrices, which plays a crucial role in implementing the PS mechanism developed by Bogomolnaia and Moulin [5]. On the other hand, our extended probabilistic serial mechanism heavily depends on the results of submodular optimization such as the integrality of the independent flow polyhedra $([8,10])$, which generalizes the Birkhoff-von Neumann theorem.

The present paper is organized as follows. In Section 2 we explain how we are motivated by the seminal paper of Bogomolnaia and Moulin [5]. Section 3 gives some definitions and preliminaries to be used later. In Section 4 we precisely describe the random assignment problem with submodular (polymatroidal and matroidal) constraints. In Section 5 we show a procedure to find an extended PS solution in the convex hull of the feasible allocations (as an expected allocation) in an ordinally efficient and fair manner. In Section 6 we examine the issue of strategy-proofness of our solution mechanism. Section 7 shows how to design a lottery efficiently to get the desired expected allocation given in Section 5. Section 8 gives concluding remarks.

The present paper is based on the authors' working papers [12] and [13].

\section{MOTIVATION AND EXAMPLES}

In this section we explain our motivation of the present paper through a series of examples of the random assignment problem and their extensions, which hopefully makes our paper more readable for those who are not very familiar with matroids and polymatroids. (Precise definitions of matroid and polymatroid will be given in Section 3.)

We are interested in obtaining a general model of the random assignment problem, fully generalized in view of the state of the art in combinatorial optimization, to which the PS mechanism of Bogomolnaia and Moulin can naturally be extended. In the history of the developments in combinatorial optimization we have a sequence of successful generalizations of the theory of combinatorial optimization as follows (see, e.g., [10, 27]).

(1) Matchings in bipartite graphs (from around 1930s).

(2) From (1) to flows in capacitated networks (from 1950s).

(3) From (1) to matroid intersection (the intersection of two matroids) (from late 1960s).

(4) From (1), (2), and (3) to polymatroid intersection (the intersection of two polymatroids) and equivalently, submodular flows, polymatroidal flows, and independent flows (intensively from late 1970s). 
(We should also mention other successful developments in the theory of combinatorial optimization such as matchings in general graphs and arborescences/branchings in directed graphs, which are not related to the motivation of our present paper.) Another important theoretical development that motivated our present research is the theory of principal partitions $[9,11]$, which is closely related to convex optimization over polymatroid base polyhedra.

When we found the seminal paper of Bogomolnaia and Moulin [5], we thought that the theory was at the stage of (1) matchings in bipartite graphs and that the probabilistic serial (PS) mechanism shown in [5] was closely related to the monotone algorithm in [9] and [10, Sec. 9.2]. Hence we felt that the results in [5] could naturally be extended to the combinatorial optimization model of (4) mentioned above. Actually, we have found that the PS mechanism of Bogomolnaia and Moulin [5] is powerful enough to have natural extensions to the combinatorial optimization model (4) of independent flows. The full extension of the PS mechanism of Bogomolnaia and Moulin [5] is worth investigating theoretically and it must also be useful for possible applications in the future if not at present.

We hope that readers would duly recognize the potential applicability of our theoretical extensions in practice through (rather toy) examples given below. The issues of ordinal efficiency, envy-freeness, and weak strategyproofness will be examined later. (Let $\mathbb{R}$ be the set of reals, $\mathbb{Z}$ the set of integers, $\mathbb{R}_{\geq 0}$ the set of nonnegative reals, $\mathbb{Z}_{\geq 0}$ the set of nonnegative integers, $\mathbb{R}_{>0}$ the set of positive reals, and $\mathbb{Z}_{>0}$ the set of positive integers.) In the following we give six illustrative examples, where the last two examples, Examples 2.5 and 2.6, cannot be modeled by using known PS extensions in the literature.

Example 2.1. Let $N=\{1,2,3\}$ be the set of three agents and suppose that there is only one good (object) $e_{0}$. Each agent wants to get the good. This can be illustrated by a graph as in Figure 1 (a). Each feasible assignment

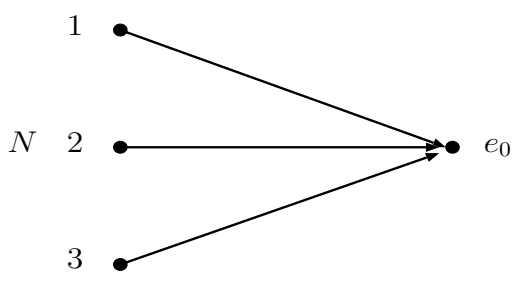

(a)

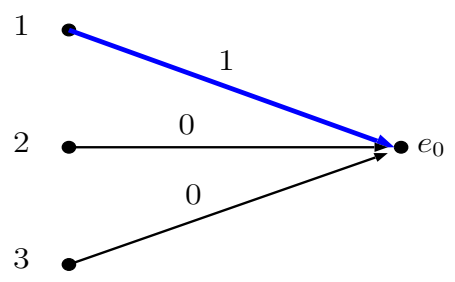

(b)

Fig. 1. (a) A simple assignment problem represented by a graph and (b) a feasible $\{0,1\}$-flow.

can be identified with a $\{0,1\}$-valued flow $\varphi$ from $N$ to $e_{0}$ of flow value 1 in the graph as in Figure $1(\mathrm{~b})$. Putting the set of arcs of the graph as $A=\left\{\left(1, e_{0}\right),\left(2, e_{0}\right),\left(3, e_{0}\right)\right\}$, the set of feasible assignments is represented by the set of $\{0,1\}$-valued vectors in $\{0,1\}^{A} \subset \mathbb{R}^{A}$ as $B=\{(1,0,0),(0,1,0),(0,0,1)\}$. Introducing a lottery with a probability distribution $p$ on $B$, we get an expected allocation $\left(p_{1}, p_{2}, p_{3}\right)$ with probability $p_{1}$ for $(1,0,0), p_{2}$ for $(0,1,0)$, and $p_{3}$ for $(0,0,1)$. The set of all expected allocations for all possible probability distributions $p$ on $B$ is the convex hull of $B$ (see Figure 2). Here we do not discuss which expected solution to choose although it is well-known that the solution $\left(\frac{1}{3}, \frac{1}{3}, \frac{1}{3}\right)$ obtained by the uniform distribution on $B$ is the fair and strategy-proof solution.

Example 2.2. Let $N=\{1,2,3\}$ and suppose that we have a set $E=\{a, b, c\}$ of three goods. Each agent $i \in N$ wants to get one of $E$ and has a preference order on $E$. Then any feasible allocation is a perfect matching in the bipartite graph $G=(N, E ; A)$ given as in Figure 3 (a). A perfect matching in $G$ can be identified with a $\{0,1\}$-valued flow $\varphi$ in $G$ as in Figure $3(\mathrm{~b})$, where a flow of value 1 enters every $i \in N$ and a flow of value 1 leaves 


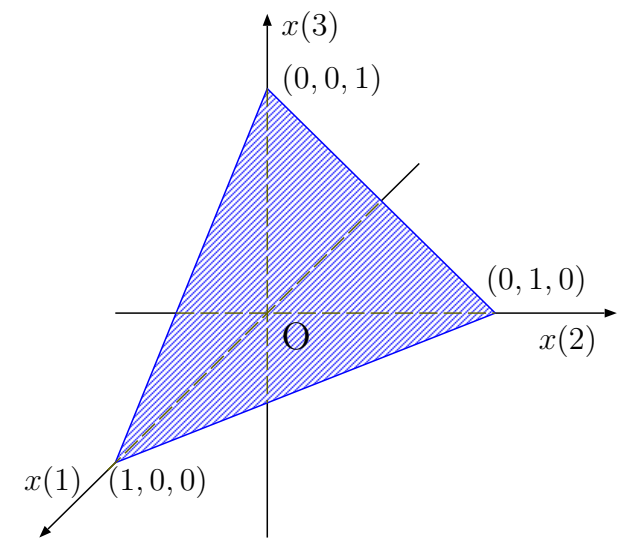

Fig. 2. The set of all expected allocations for Example 2.1.

every $e \in E$. Let $\mathbf{M}$ be the set of all feasible allocations, i.e., the set of all perfect matchings of $G$. We regard every perfect matching in $G$ as a $\{0,1\}$-vector in $\mathbb{R}^{A}$. Then every lottery on $\mathbf{M}$ determines an expected allocation that is a vector in the convex hull $\operatorname{Conv}(\mathbf{M})$ of $\mathbf{M}$ in $\mathbb{R}^{A}$. Note that all the possible lotteries on $\mathbf{M}$ generate exactly the points of the convex hull $\operatorname{Conv}(\mathbf{M})$ in $\mathbb{R}^{A}$, which is known as the Birkhoff-von Neumann polytope of bi-stochastic matrices. Now the problem is how to determine a solution, a desired expected allocation in $\operatorname{Conv}(\mathbf{M})$ and to design a lottery that realizes the solution. This is exactly the model treated by Bogomolnaia and Moulin [5].

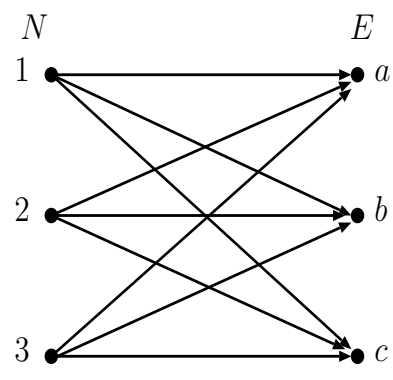

(a)

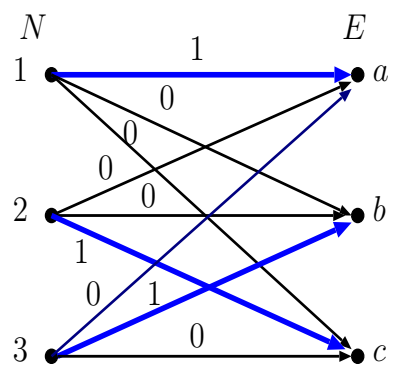

(b)

Fig. 3. (a) A bipartite graph $G=(N, E ; A)$ and (b) a perfect matching as a feasible flow in $G$ for Example 2.2.

Example 2.3. Let $N=\{1,2,3\}$. Now suppose that we have a set $E=\{a, b, c, d\}$ of four goods and that because of some budget constraint only two goods from $E$ can be offered to the agents, while each agent wants to get one good from $E$ and has a preference order on $E$. Every feasible allocation for the present problem can be regarded as a $\{0,1\}$-valued flow $\varphi$ of flow value two from $N$ to $E$ in the bipartite graph $G=(N, E ; A)$ (given as in Figure 4) such that a flow of value 0 or 1 enters every $i \in N$ and a flow of value 0 or 1 leaves every $e \in E$. Figure 4 shows a feasible allocation that assigns good $c$ to agent 1 and good $d$ to agent 2 .

Here the family $\mathcal{B}$ of available two-element subsets of $E$ is a typical example of a matroid base family of a uniform matroid of rank two (the precise definition will be given later). It should also be noted that this matroidal 


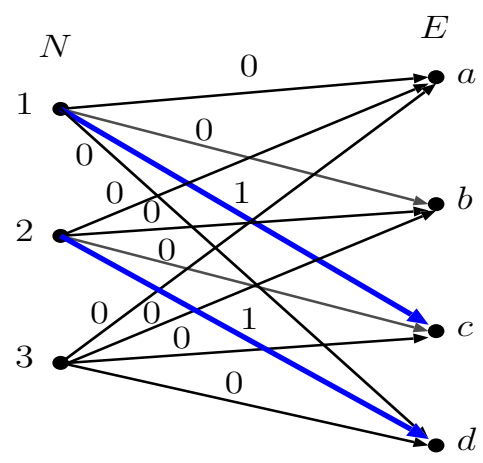

Fig. 4. A feasible flow in a bipartite graph $G=(N, E ; A)$ for Example 2.3.

constraint can be treated as a network flow model as in Figure 5, where every feasible allocation can be regarded as a $\{0,1\}$-valued flow $\varphi$ in the graph such that a flow of value 0 or 1 enters every $i \in N, \varphi$ satisfies the flow conservation law at every internal vertex $e \in E$, and a flow of value two leaves the exit $t$, where every arc capacity is equal to one.

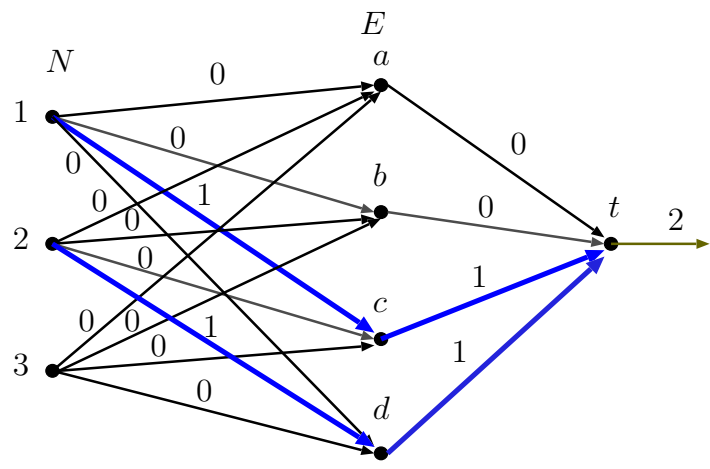

Fig. 5. (I) A network-flow representation and a feasible flow for Example 2.3.

Example 2.4. Similarly as in Example 2.3 we may also treat, as a matroidal constraint, the case when at most one good from $\{a, b\}$, at most one good from $\{c, d\}$, and two goods in total are available. This is a matroidal constraint represented by the direct sum of rank-one uniform matroid on $\{a, b\}$ and that on $\{c, d\}$. As can easily be seen, this can also be formulated as a network flow model similarly as in Example 2.3 (see Figure 6). Actually these types of constraints here and in Examples 2.3 are considered by E. Budish et al. [6] as a laminar constraint ${ }^{1}$, which can be expressed by a directed tree from the set $E$ of leaves toward the root (exit) $t$ with arcs having appropriate capacities. It should further be noted that beyond the laminar constraints represented by directed

${ }^{1}$ They call a laminar constraint a hierarchy. A family $\mathcal{F} \subseteq 2^{E}$ is called laminar if for all $X, Y \in \mathcal{F}$ we have $X \subseteq Y, Y \subseteq X$, or $X \cap Y=\emptyset$. 
trees as considered by Budish et al. [6], we can consider any directed network from $E$ to exit $t$, which represents a (poly)matroidal constraint of network type ${ }^{2}$.

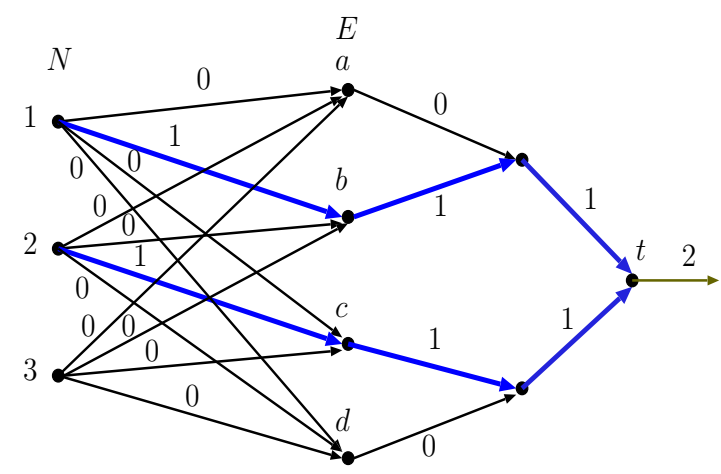

Fig. 6. (II) A network-flow representation and a feasible flow for Example 2.4.

Moreover, it should be noted that the convex hull of all the $\{0,1\}$-valued flows is given by the set of real-valued flows in the associated network, due to the integrality property of flows in networks with integral capacity functions. This gives a basis for constructing a lottery to realize a desired solution.

Example 2.5. Let $N=\{1,2,3\}$ and $E=\{a, b, c, d\}$ as in Examples 2.3 and 2.4. Suppose that the set $\mathcal{B}$ of available subsets of $E$ is that of all two-element subsets of $E$ but $\{a, b\}$, i.e.,

$$
\mathcal{B}=\{X|X \subset E,| X \mid=2, X \neq\{a, b\}\} .
$$

This is a graphic matroid, which can be represented by a graph $H=(V, E)$ with $E$ being the edge set as shown in Figure 7. Here the feasible sets in $\mathcal{B}$ are exactly the edge subsets that form spanning trees of $H$.

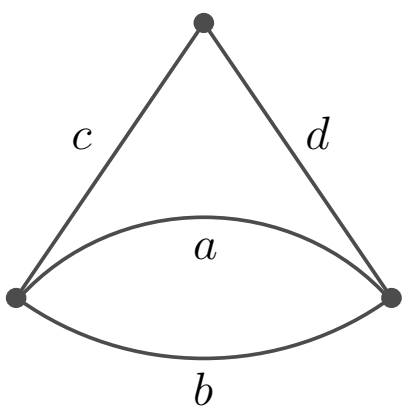

Fig. 7. A graph $H=(V, E)$ with edge set $E=\{a, b, c, d\}$.

We can consider any matroidal constraints on the set of available subsets of goods.

${ }^{2}$ Budish et al. [6] considered $\{0,1\}$-valued flows in bipartite networks with laminar constraints on both sides of vertex sets $N$ and $E$ (called a bihierarchy. This model is a special case of the independent flow model with polymatroids of network type on both sets of entrances and of exits, which will precisely be defined in Section 3. 
Example 2.6. Let $N=\{1,2,3\}$ and $E=\{a, b, c, d\}$. Now we suppose that $E$ is the set of types of goods and there exist multi-units of each good $e \in E$. As an example consider a polymatroidal constraint of network type shown in Figure 8. In Figure 8 each arc is given an integer capacity, where $+\infty$ is regarded as a sufficiently large

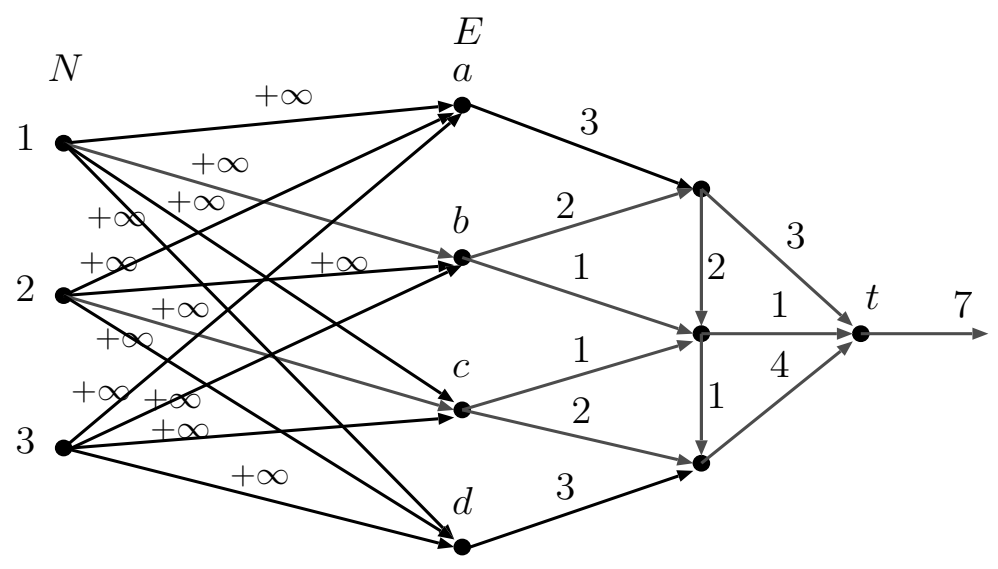

Fig. 8. A polymatroidal constraint of network type (an integer attached to each arc denotes the arc capacity).

integer. We suppose that each agent $i \in N$ has a demand $d(i)$ (the total number of goods of any types) given by $d(1)=3, d(2)=2$, and $d(3)=3$ and the total amount of available goods (of any types) is equal to 7 . Then every feasible allocation corresponds to an integral flow $\varphi$ in the network such that (1) the flow value entering each $i \in N$ is at most $d(i)$, (2) the flow value leaving the exit $t$ is equal to 7, and (3) $\varphi$ satisfies the capacity constraint of every arc and the flow conservation at every internal vertex. An integral feasible flow $\varphi$ is shown in Figure 9. An

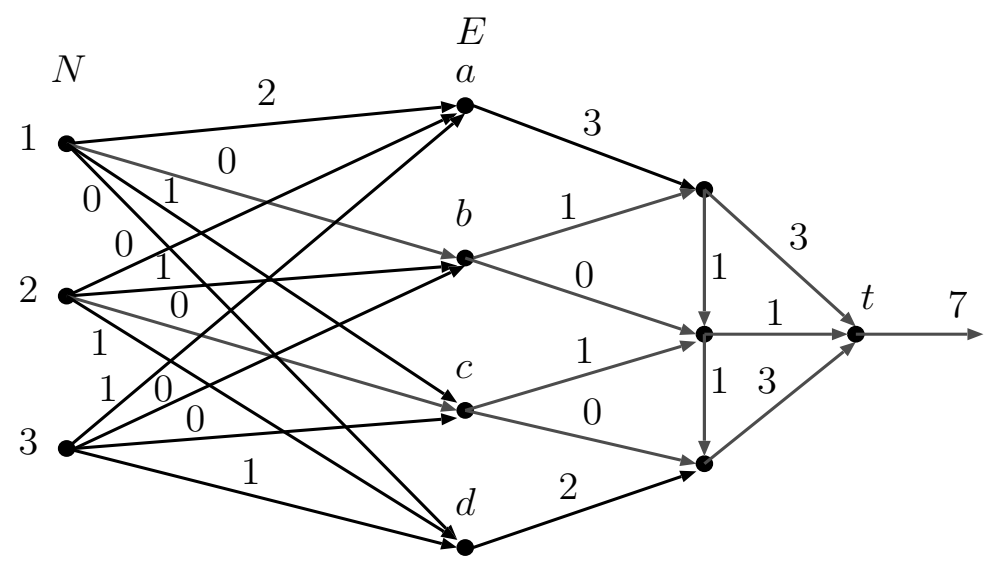

Fig. 9. An integral flow $\varphi$ in the network (an integer attached to each arc denotes the arc flow value), where agent 1 receives two copies of good $a$ and one copy of good $c$.

integral $N \times E$ matrix $(\varphi(i, e) \mid i \in N, e \in E)$ gives a feasible allocation. The set $\mathbf{B}$ of all available good vectors is 
the set of vectors $x \in \mathbb{Z}_{\geq 0}^{E}$ defined by $x(e)=\sum_{i \in N} \varphi(i, e)(e \in E)$ for all feasible integral flows $\varphi$ in the network. Set $\mathbf{B}$ is the set of integral bases of a polymatroid of network type, represented by the subnetwork connecting $E=\{a, b, c, d\}$ with exit $t$. Besides such polymatroids of network type we can consider any integral polymatroidal set (an integral base polyhedron) of available good vectors in $\mathbb{Z}_{\geq 0}^{E}$.

\section{DEFINITIONS AND PRELIMINARIES}

In this section we give definitions of some concepts from the theory of matroids and polymatroids and also give preliminary lemmas and theorems to be used in the following (see, e.g., $[10,25,27,30]$ ).

Let $E$ be a nonempty finite set. For any subset $X \subseteq E$ denote by $\chi_{X}$ the characteristic vector of $X$ in $\mathbb{R}^{E}$, i.e., $\chi_{X}(e)=1$ for $e \in X$ and $\chi_{X}(e)=0$ for $e \in E \backslash X$. We also write $\chi_{e}$ instead of $\chi_{\{e\}}$ for $e \in E$.

A pair $(E, \rho)$ of set $E$ and a function $\rho: 2^{E} \rightarrow \mathbb{R}_{\geq 0}$ is called a polymatroid [7] if the following three conditions hold (also see $[10,27,30])$.

(1) $\rho(\emptyset)=0$.

(2) For any $X, Y \in 2^{E}$ with $X \subseteq Y$ we have $\rho(X) \leq \rho(Y)$.

(3) For any $X, Y \in 2^{E}$ we have $\rho(X)+\rho(Y) \geq \rho(X \cup Y)+\rho(X \cap Y){ }^{3}$

The function $\rho$ is called the rank function of the polymatroid $(E, \rho)$. We assume $\rho(E)>0$ in the sequel.

For a given polymatroid $(E, \rho)$, let $\mathrm{B}(\rho)\left(\subseteq \mathbb{R}^{E}\right)$ be the base polyhedron of the polymatroid (see [10]), which is given by

$$
\mathrm{B}(\rho)=\left\{x \in \mathbb{R}^{E} \mid \forall X \subset E: x(X) \leq \rho(X), x(E)=\rho(E)\right\},
$$

where for any $X \subseteq E$ we define $x(X)=\sum_{e \in X} x(e)$. It should be noted that $\mathrm{B}(\rho) \subseteq \mathbb{R}_{\geq 0}^{E}$. Moreover, we have the following relation between the rank function $\rho$ and the base polyhedron $\mathrm{B}(\rho)$.

$$
\rho(X)=\max \{x(X) \mid x \in \mathrm{B}(\rho)\} \quad(\forall X \subseteq E) .
$$

Equations (2) and (3) determine a one-to-one correspondence between the set of rank functions $\rho: 2^{E} \rightarrow \mathbb{R}$ and the set of base polyhedra $\mathrm{B}(\rho) \subset \mathbb{R}^{E}$. It should be noted that the concept of the base polyhedron of a polymatroid is equivalent to that of the core of a convex game due to Shapley [29].

Also consider the lower hereditary closure of the base polyhedron $\mathrm{B}(\rho)$ given by

$$
\mathrm{P}(\rho)=\left\{x \in \mathbb{R}^{E} \mid \forall X \subseteq E: x(X) \leq \rho(X)\right\},
$$

which is called the submodular polyhedron associated with polymatroid $(E, \rho)$. The polytope $\mathrm{P}_{(+)}(\rho) \equiv \mathrm{P}(\rho) \cap \mathbb{R}_{\geq 0}^{E}$ is called the independence polytope of polymatroid $(E, \rho)$ and each vector in $\mathrm{P}_{(+)}(\rho)$ is called an independent vector. Note that relation (3) holds when we replace $\mathrm{B}(\rho)$ by $\mathrm{P}(\rho)$ (respectively, $\mathrm{P}_{(+)}(\rho)$ ) in (3), as well, and $\mathrm{P}(\rho)$ uniquely determines the rank function $\rho: 2^{E} \rightarrow \mathbb{R}$ (see Figure 10).

Given a vector $x \in \mathrm{P}(\rho)$, a subset $X$ of $E$ is called tight for $x$ (or $x$-tight for short) if we have $x(X)=\rho(X)$, and there exists a unique maximal $x$-tight set, denoted by sat $(x)$, which is equal to the union of all tight sets for $x$. We also have

$$
\operatorname{sat}(x)=\left\{e \in E \mid \forall \alpha>0: x+\alpha \chi_{e} \notin \mathrm{P}(\rho)\right\},
$$

which is the set of elements $e \in E$ for which we cannot increase $x(e)$ without leaving $\mathrm{P}(\rho)$. Moreover, for $x \in \mathrm{P}(\rho)$ and $e \in \operatorname{sat}(x)$ define

$$
\operatorname{dep}(x, e)=\left\{e^{\prime} \in E \mid \exists \alpha>0: x+\alpha\left(\chi_{e}-\chi_{e^{\prime}}\right) \in \mathrm{P}(\rho)\right\} .
$$

The following fact is fundamental in the theory of polymatroids and submodular functions.

\footnotetext{
${ }^{3}$ A set function satisfying these inequalities is called a submodular function and the negative of a submodular function is called a supermodular function. A function that is submodular and at the same time supermodular is called a modular function.
} 

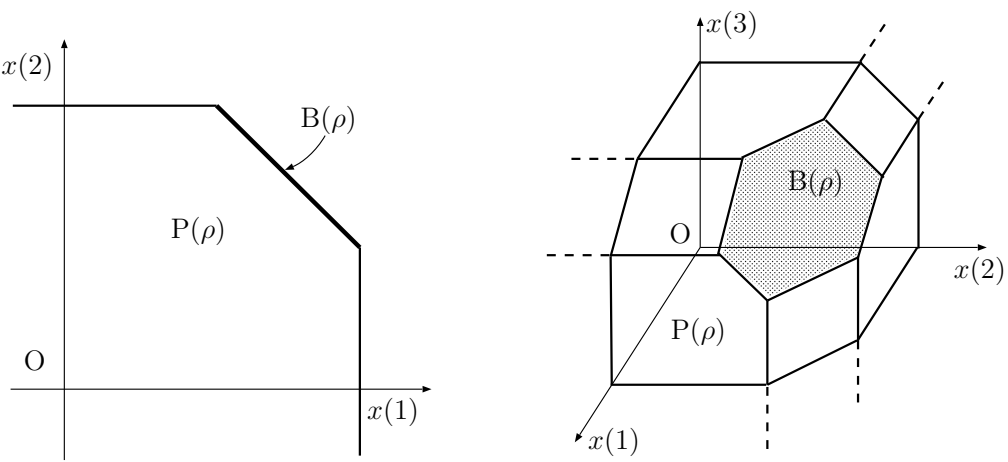

Fig. 10. Base polyhedra $\mathrm{B}(\rho)$ and submodular polyhedra $\mathrm{P}(\rho)$.

Lemma 3.1. Given any $x \in \mathrm{P}(\rho)$ and $X, Y \subseteq E$, if we have $x(X)=\rho(X)$ and $x(Y)=\rho(Y)$, then $x(X \cup Y)=\rho(X \cup Y)$ and $x(X \cap Y)=\rho(X \cap Y)$. That is, the set of $x$-tight sets is closed with respect to the set union and intersection.

Because of this fact $\operatorname{sat}(x)$ for $x \in \mathrm{P}(\rho)$ is the unique maximal $x$-tight set and $\operatorname{dep}(x, e)$ for $e \in \operatorname{sat}(x)$ is the unique minimal $x$-tight set that includes $e$. (See [10] for more details about these concepts and related facts.)

If the rank function $\rho$ of a polymatroid $(E, \rho)$ is integer-valued and satisfies $\rho(\{e\}) \leq 1$ for all $e \in E$, then $(E, \rho)$ is called a matroid (see, e.g., $[25,30])$. When $(E, \rho)$ is a matroid, define

$$
\mathcal{I}=\{X \subseteq E|| X \mid=\rho(X)\}, \quad \mathcal{B}=\{X \in \mathcal{I}|| X \mid=\rho(E)\} .
$$

Each $I \in \mathcal{I}$ is called an independent set and $\mathcal{I}$ is the family of independent sets of $(E, \rho)$. Each $B \in \mathcal{B}$ is called a base and $\mathcal{B}$ is called the family of bases and consists of all maximal independent sets of $(E, \rho)$ (maximal with respect to set inclusion). Since each of $\rho, \mathcal{B}$ and $\mathcal{I}$ uniquely determines the matroid on $E$, we also denote by $(E, \mathcal{B})$ (or $(E, \mathcal{I}))$ the matroid $(E, \rho)$.

For any polymatroid $(E, \rho)$ with an integer-valued rank function $\rho$ define

$$
\mathrm{B}_{\mathbb{Z}}(\rho)=\mathrm{B}(\rho) \cap \mathbb{Z}^{E}, \quad \mathrm{P}_{\mathbb{Z}}(\rho)=\mathrm{P}(\rho) \cap \mathbb{Z}^{E} .
$$

The following is well known (see, e.g., [10]).

THEOREM 3.2. When $(E, \rho)$ is a polymatroid with an integer-valued rank function $\rho, \mathrm{B}(\rho)($ resp. $\mathrm{P}(\rho))$ is the convex hull of $\mathrm{B}_{\mathbb{Z}}(\rho)$ (resp. $\left.\mathrm{P}_{\mathbb{Z}}(\rho)\right)$. Moreover, when $(E, \rho)$ is a matroid, $\mathrm{B}_{\mathbb{Z}}(\rho)\left(\right.$ or $\left.\mathrm{P}_{(+)}(\rho) \cap \mathbb{Z}^{E}\right)$ is exactly the set of all the characteristic vectors of bases (or independent sets) of matroid $(E, \rho)$.

For a polymatroid $(E, \rho)$ and a nonempty subset $F \subseteq E$ define $\rho^{F}: 2^{F} \rightarrow \mathbb{R}$ by $\rho^{F}(X)=\rho(X)$ for all $X \subseteq F$. Then we have a polymatroid $\left(F, \rho^{F}\right)$, which is called a reduction of $(E, \rho)$ by $F$ (or restriction of $(E, \rho)$ to $F$ ). Moreover, define $\rho_{F}: 2^{E \backslash F} \rightarrow \mathbb{R}$ by $\rho_{F}(X)=\rho(F \cup X)-\rho(F)$ for all $X \subseteq E \backslash F$. Then $\left(E \backslash F, \rho_{F}\right)$ is a polymatroid, called a contraction of $(E, \rho)$ by $F$. For any nonempty $F_{1}, F_{2} \subseteq E$ with $F_{1} \subset F_{2}$ we put $\rho_{F_{1}}^{F_{2}}=\left(\rho^{F_{2}}\right)_{F_{1}}$, which defines a polymatroid $\left(F_{2} \backslash F_{1}, \rho_{F_{1}}^{F_{2}}\right)$, called a minor of $(E, \rho)$.

For any vector $x \in \mathbb{R}^{E}$ and any nonempty set $A \subseteq E$ define $x^{A} \in \mathbb{R}^{A}$ by $x^{A}(e)=x(e)$ for all $e \in A$. We have the following lemma, which will be used in Section 6.1.1.

Lemma 3.3. Let $\mathbf{P}=(E, \rho)$ be a polymatroid. For any vectors $x, y \in \mathrm{P}(\rho)$ satisfying $x(e) \geq y(e)(\forall e \in E \backslash \operatorname{sat}(x))$, we have $\operatorname{sat}(x) \supseteq \operatorname{sat}(y)$. 
(Proof) Suppose that we are given vectors $x, y \in \mathrm{P}(\rho)$ satisfying $x(e) \geq y(e)(\forall e \in E \backslash \operatorname{sat}(x))$. Increase the values of $y(e)$ for all $e \in \operatorname{sat}(x)$ as much as possible while keeping the vector within $\mathrm{P}(\rho)$, and let us denote by $y^{\prime}$ the resulting vector in $\mathrm{P}(\rho)$. Then, we have sat $\left(y^{\prime}\right) \supseteq \operatorname{sat}(x)$. Letting $A=\operatorname{sat}(x)$ and $B=\operatorname{sat}\left(y^{\prime}\right)$, if $\rho(A)>y^{\prime}(A)$, then

$$
\rho(B)-\rho(A)<y^{\prime}(B)-y^{\prime}(A) \leq x(B)-x(A) .
$$

Since $\rho(A)=x(A)$, this implies $\rho(B)<x(B)$, a contradiction. Hence $\rho(A)=y^{\prime}(A)$ and $\left(y^{\prime}\right)^{A}$ and $x^{A}$ are bases of $\mathbf{P} \cdot A=\left(A, \rho^{A}\right)$, the restriction of $\mathbf{P}$ to $A$. Hence $\left(y^{\prime}\right)^{E \backslash A} \in \mathrm{P}\left(\rho_{A}\right)$, where $\rho_{A}$ is the rank function of the contraction $\left(E \backslash A, \rho_{A}\right)$ of $\mathbf{P}$ by $A$. Since $x(e) \geq y^{\prime}(e)(\forall e \in E \backslash A)$ and $\operatorname{sat}(x)=A$, we have $\operatorname{sat}(y) \subseteq \operatorname{sat}\left(y^{\prime}\right) \subseteq \operatorname{sat}(x)=A$.

Simple examples of polymatroids, some of which has already appeared in Section 2, are given as follows.

Uniform matroids: For a positive integer $k \leq m(=|E|)$ every subset of cardinality $k$ of $E$ is exactly a base, i.e.,

$$
\mathcal{B}=\{X|X \subseteq E,| X \mid=k\} .
$$

When $k=m(=|E|), \mathcal{B}$ consists of only one base $E$, which is the unique available set of goods as considered in the literature for the ordinary random assignment problem.

Graphic matroids: For a connected graph $G=(V, E)$ with a vertex set $V$ and an edge set $E$ every edge subset that forms a spanning tree of $G$ is exactly a base, i.e., $(E, \mathcal{B})$ is the graphic matroid represented by graph $G=(V, E)$ with $\mathcal{B}$ being the family of edge sets of spanning trees. Note that for any $X \subseteq E$ the value of $\rho(X)$ is equal to the maximum size of cycle-free subsets of $X$ in $G$.

Symmetric polymatroids: Let $g: \mathbb{R} \rightarrow \mathbb{R}$ be a nondecreasing concave function with $g(0)=0$. Define $\rho: 2^{E} \rightarrow \mathbb{R}$ by $\rho(X)=g(|X|)$. Then $(E, \rho)$ is a polymatroid. Note that the concavity of $g$ corresponds to the law of diminishing marginal utility in economics.

Linear polymatroids: Let $V$ be a vector space. Let $E$ be a nonempty finite set and for each $e \in E$ let $F_{e}$ be a finite set of vectors in $V$. Define $\rho: 2^{E} \rightarrow \mathbb{R}$ by $\rho(X)=\operatorname{rank}\left(\bigcup_{e \in X} F_{e}\right)$ for all $X \subseteq E$. Then $(E, \rho)$ is a polymatroid with the integer-valued rank function $\rho$.

Polymatroids of multi-terminal network flows ([23],[10, Sec 2.2]; also see [9, 15]): Let $\mathcal{N}=(G=(V, A), s, T, c)$ be a network, where $G=(V, A)$ is a graph with a vertex set $V$ and an arc set $A, s \in V$ is a source, $T \subset V \backslash\{s\}$ is a set of sink terminals, and $c: A \rightarrow \mathbb{R}_{>0}$ is a capacity function of the network. We suppose that there exists no arc leaving $T$. A function $\varphi: A \rightarrow \mathbb{R}_{\geq 0}$ is called a feasible flow in $\mathcal{N}$ if it satisfies the capacity constraints

$$
0 \leq \varphi(a) \leq c(a) \quad(\forall a \in A)
$$

and the flow conservation constraints

$$
\partial \varphi(v)=0 \quad(\forall v \in V \backslash(\{s\} \cup T)),
$$

where the boundary $\partial \varphi: V \rightarrow \mathbb{R}$ of flow $\varphi$ is defined by

$$
\partial \varphi(v)=\sum_{(v, w) \in A} \varphi(v, w)-\sum_{(w, v) \in A} \varphi(w, v) \quad(\forall v \in V) .
$$

Also define the out-flow $\partial^{-} \varphi: T \rightarrow \mathbb{R}_{\geq 0}$ of $\varphi$ by

$$
\partial^{-} \varphi(v)=\sum_{(w, v) \in A} \varphi(w, v)(=-\partial \varphi(v)) \quad(\forall v \in T) .
$$

Then the set of out-flows $\partial^{-} \varphi \in \mathbb{R}_{\geq 0}^{T}$ of all feasible flows $\varphi$ in $\mathcal{N}$ is the independence polytope, in $\mathbb{R}_{\geq 0}^{T}$, of a polymatroid $(T, \rho)$ on $T$. For any $X \subseteq T$ the value of rank $\rho(X)$ is equal to the maximum flow value from $s$ to $X$ in $\mathcal{N}$. (It should be noted that polymatroids of network type have appeared in Examples 2.3-2.6 in Section 2, where arcs of the representing networks are reoriented.) 
When the underlying graph $G=(V, A)$ is a star such that $V=\{s\} \cup T$ and $A=\{(s, t) \mid t \in T\}$, we have a polymatroid on $T$ with a modular rank function $\rho$ such that $\rho(X)=\sum_{v \in X} c(s, v)$ for all $X \subseteq T$. Any polymatroid of this kind has a unique base and vice versa.

Consider a capacitated network $\mathcal{N}=\left(G=(V, A), S^{+}, S^{-}, c,\left(S^{+}, \rho^{+}\right),\left(S^{-}, \rho^{-}\right)\right)$with polymatroids on sets $S^{+}, S^{-} \subset V$. Here $G$ is the underlying graph with vertex set $V$ and $\operatorname{arc} \operatorname{set} A$, and $S^{+}$and $S^{-}$are disjoint subsets of $V$ and are, respectively, the set of sources (entrances) and that of sinks (exits). Furthermore, we have a capacity function $c: A \rightarrow \mathbb{R}_{\geq 0}$ and a pair of polymatroids $\left(S^{+}, \rho^{+}\right)$and $\left(S^{-}, \rho^{-}\right)$. A function $\varphi: A \rightarrow \mathbb{R}$ is called an independent flow in $\mathcal{N}$ if it satisfies

$$
\begin{aligned}
& 0 \leq \varphi(a) \leq c(a) \quad(\forall a \in A), \\
& \partial \varphi(v)=0 \quad\left(\forall v \in V \backslash\left(S^{+} \cup S^{-}\right)\right), \\
& \partial^{+} \varphi \in \mathrm{P}_{(+)}\left(\rho^{+}\right), \quad \partial^{-} \varphi \in \mathrm{P}_{(+)}\left(\rho^{-}\right),
\end{aligned}
$$

where $\partial^{ \pm} \varphi: S^{ \pm} \rightarrow \mathbb{R}$ are defined by $\partial^{+} \varphi(v)=\partial \varphi(v)$ for all $v \in S^{+}$and $\partial^{-} \varphi(v)=-\partial \varphi(v)$ for all $v \in S^{-}$. Note that (13) is the flow capacity constraint for each arc, (14) the flow conservation constraint on each internal vertex, and (15) the polymatroidal boundary constraints on the entrance set $S^{+}$and the exit set $S^{-}$. The value $\partial^{+} \varphi\left(S^{+}\right)\left(=\partial^{-} \varphi\left(S^{-}\right)\right)$is called the flow value (or simply value) of the independent flow $\varphi$. We may also consider a cost function $\gamma: A \rightarrow \mathbb{R}$, which gives a problem of finding a minimum-cost independent flow in $\mathcal{N}$. This is called the independent flow problem [8] and is equivalent to what is called the submodular flow problem (see [10]).

We have the following integrality theorem $([8,10])$, which plays a crucial role in validating our approach based on the PS mechanism of Bogomolnaia and Moulin [5].

Theorem 3.4. Let $P^{*} \subset \mathbb{R}^{A}$ be the set of all independent flows $\varphi$ in network $\mathcal{N}=\left(G=(V, A), S^{+}, S^{-}, c,\left(S^{+}, \rho^{+}\right)\right.$, $\left.\left(S^{-}, \rho^{-}\right)\right)$. If $c$ and $\rho^{ \pm}$are integer-valued, then $P^{*}$ is an integral polytope, i.e., $P^{*}$ is a convex polytope such that every extreme point of $P^{*}$ is an integral vector. Moreover, the same integrality property also holds if we consider the set of independent flows of fixed integral value $\partial^{+} \varphi\left(S^{+}\right)\left(=\partial^{-} \varphi\left(S^{-}\right)\right)$.

\section{DESCRIPTION OF THE RANDOM ASSIGNMENT PROBLEM}

Now we give a precise definition of the random assignment problem with polymatroidal constraints and later examine the problem with matroidal constraints as a special case.

\subsection{Model description}

Let $N=\{1,2, \cdots, n\}$ be a set of agents and $E$ be a set of goods. Each good $e \in E$ should be considered as a type of good and the number of available good $e$ can be more than one (see Example 2.6). Each agent $i \in N$ wants to obtain a certain amount of goods of any types, denoted by $d(i) \in \mathbb{Z}_{>0}$, at most in total. We refer to $d(i)$ as the demand upper bound of agent $i$. The vector $d=(d(i) \mid i \in N) \in \mathbb{Z}_{>0}^{N}$ is called the demand vector. For each $i \in N$ and $e \in E$ let $x^{i}(e)$ be the number of copies of good $e$ that agent $i$ obtains. Then we must have

$$
x^{i}(E) \equiv \sum_{e \in E} x^{i}(e) \leq d(i)
$$

for every agent $i \in N$. Let $\mathbf{B} \subseteq \mathbb{Z}_{\geq 0}^{E}$ be the set of all available vectors of goods in the market that is given by $\mathbf{B}=\mathrm{B}_{\mathbb{Z}}(\rho)$ for a polymatroid $(E, \rho)$ with an integer-valued rank function $\rho$ (see (8)). Since the sum of vectors $\sum_{i \in N} x^{i}$ must be available in the market, we have the following constraint.

$$
\sum_{i \in N} x^{i} \in \mathrm{B}_{\mathbb{Z}}(\rho) .
$$

We assume that $\rho(E) \leq d(N)$. 
Define $\mathrm{A} \subseteq \mathbb{Z}_{\geq 0}^{N \times E}$ to be the set of all functions $\varphi: N \times E \rightarrow \mathbb{Z}_{\geq 0}$ such that vectors given by $x^{i}=(\varphi(i, e) \mid e \in E)$ for all $i \in N$ satisfy (16) and (17). Every $\varphi \in$ A determines a feasible allocation $x^{i}=(\varphi(i, e) \mid e \in E)$ for each agent $i \in N$. Note that a function $\varphi: N \times E \rightarrow \mathbb{Z}_{\geq 0}$ is identified with an $N \times E$ matrix $P=(\varphi(i, e) \mid i \in N, e \in E)$, and $x^{i}$ is with the $i$ th row of $P$ for each $i \in N$.

Consider an independent-flow network $\mathcal{N}=\left(G=\left(S^{+}, S^{-} ; A\right), c,\left(S^{+}, \rho^{+}\right),\left(S^{-}, \rho^{-}\right)\right)$, where $S^{+}=N, S^{-}=E$, $G=\left(S^{+}, S^{-} ; A\right)$ is a complete bipartite graph with vertex bi-partition $\left(S^{+}, S^{-}\right)$and $\operatorname{arc} \operatorname{set} A=S^{+} \times S^{-}, c(a)=+\infty$ (a sufficiently large positive integer) for all $a \in A,\left(S^{-}, \rho^{-}\right)$is an integral polymatroid with rank function $\rho^{-}=\rho$ appearing in (17), and $\left(S^{+}, \rho^{+}\right)$is a polymatroid with a (modular) rank function $\rho^{+}$given by $\rho^{+}(X)=d(X)$ for all $X \subseteq S^{+}=N{ }^{4}$ For simplicity we also denote the present independent-flow network by $\mathcal{N}=(N, E, d,(E, \rho))$ (see Figure 11). Then from Theorem 3.2 we can easily see the following.

Lemma 4.1. The set $\mathrm{A}$ is exactly the set of integer-valued independent flows $\varphi: S^{+} \times S^{-} \rightarrow \mathbb{Z}_{\geq 0}$ of value $\partial^{+} \varphi(N)=\partial^{-} \varphi(E)=\rho(E)$ in network $\mathcal{N}=(N, E, d,(E, \rho))$.

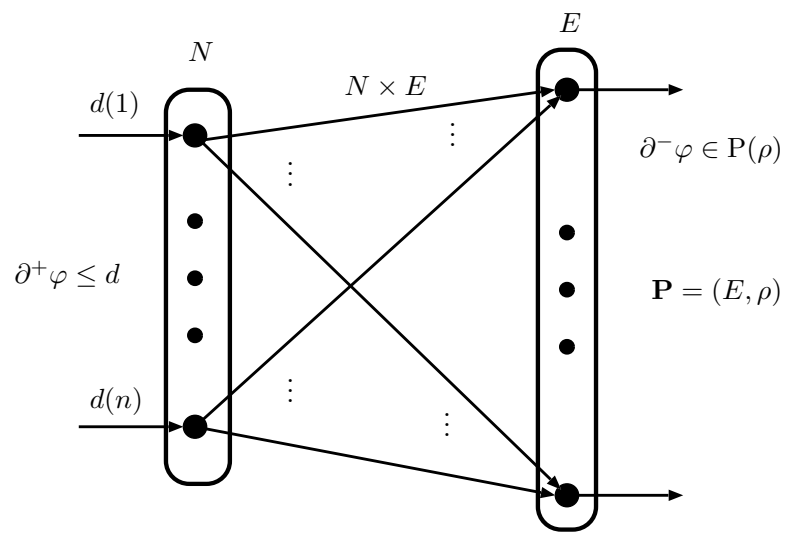

Fig. 11. An independent-flow network $\mathcal{N}$.

Because of Theorem 3.4 and Lemma 4.1 we also have the following.

Corollary 4.2. The set of all (real-valued) independent flows $\varphi$ of value $\rho(E)$ in $\mathcal{N}=(N, E, d,(E, \rho))$ is the convex hull $\operatorname{Conv}(\mathrm{A})$ of all integer-valued independent flows of value $\rho(E)$ in $\mathcal{N}$.

\subsection{Ordinal preference and stochastic dominance relation}

We suppose that each agent $i \in N$ has an ordinal preference $>_{i}$ over set $E$ of types of goods ${ }^{5}$, which is a linear ordering of $E$. Let agent $i$ 's preference be given by

$$
L^{i}: e_{1}^{i}>_{i} e_{2}^{i}>_{i} \cdots>_{i} e_{m}^{i}
$$

\footnotetext{
${ }^{4}$ We may be tempted to consider a general polymatroid as $\left(S^{+}, \rho^{+}\right)$on the set $S^{+}=N$ of agents instead of a modular rank function $\rho^{+}$ Though the PS mechanism of Bogomolnaia and Moulin can formally be extended to such a general model mathematically, we cannot adapt our arguments to validate the assertions given in the sequel, especialy in Section 6. However, because of Theorem 3.2 the procedure shown in Section 7 for designing a lottery to realize any given expected allocation can be adapted even for such a general model (cf. the model with a bihierarchy structure in [6]).

${ }^{5}$ We often call a type of good $e \in E$ as a good $e$ in the sequel, where there is no possibility of confusion.
} 
where $\left\{e_{1}^{i}, e_{2}^{i}, \cdots, e_{m}^{i}\right\}=E^{6}$ and $e_{1}^{i}$ is the most favorite (type of) good for agent $i$. Let $\mathcal{L}$ be the profile of preferences $L^{i}(i \in N)$.

Since we must make a decision on how to allocate goods in a fair manner without money, we may consider a lottery, which is represented by a probability distribution $p$ over A, i.e., $p: \mathbf{A} \rightarrow \mathbb{R}_{\geq 0}$ satisfying $\sum_{\varphi \in \mathrm{A}} p(\varphi)=1$. Then the expected allocation of goods is given by

$$
\mathbf{E}\{\varphi\}=\sum_{\varphi \in \mathrm{A}} p(\varphi) \varphi
$$

where precisely speaking, the left-hand side is the expectation of a random variable $\varphi$ with its probability distribution $p$ on A while $\varphi$ appearing on the right-hand side is a variable taking on values of A. It should be noted that the set of all expected allocations $\mathbf{E}\{\varphi\}$ of (19) for all possible probability distributions $p$ is exactly the convex hull $\operatorname{Conv}(\mathrm{A})$ of A and that every lottery picks up a point from among $\operatorname{Conv}(\mathrm{A})$.

In the following we use $N \times E$ matrices $P$ to express expected allocations $\varphi \in \operatorname{Conv}(\mathrm{A})$ by identifying $\varphi$ with $P=(\varphi(i, e) \mid i \in N, e \in E)$, which is often employed in the literature. So we may write $P \in \operatorname{Conv}(\mathrm{A})$, for example. When $\varphi$ corresponds to $P, \varphi$ is sometimes written as $\varphi_{P}$.

An efficient and fair expected allocation will be found with respect to the stochastic dominance relation (sddominance relation for short) $\geq_{i}^{\text {sd }}$ for each agent $i \in N$ on expected allocations defined as follows. Recall that we are given a preference profile $\mathcal{L}=\left(L^{i} \mid i \in N\right)$ of (18). For any $P, Q \in \operatorname{Conv}(\mathrm{A})$, putting $P_{i}=(P(i, e) \mid e \in E)$ and $Q_{i}=(Q(i, e) \mid e \in E)$ for all $i \in N$, we define

$$
P_{i} \geq_{i}^{\text {sd }} Q_{i} \quad \Longleftrightarrow \quad \forall \ell \in\{1, \cdots, m\}: \sum_{k=1}^{\ell} P\left(i, e_{k}^{i}\right) \geq \sum_{k=1}^{\ell} Q\left(i, e_{k}^{i}\right) .
$$

We say an expected allocation $P$ is $s d$-dominated by $Q$ if we have $Q_{i} \geq_{i}^{\text {sd }} P_{i}$ for all $i \in N$ and $P \neq Q$. We say that $P$ is ordinally efficient if $P$ is not sd-dominated by any other expected allocation in Conv(A) (cf. [5]).

Also, we say an expected allocation $P$ is normalized envy-free ([18]) with respect to preference profile $\mathcal{L}=$ $\left(L^{i} \mid i \in N\right)$ of (18) if we have

$$
\frac{1}{d(i)} P_{i} \geq_{i}^{\mathrm{sd}} \frac{1}{d(j)} P_{j} \quad(\forall i, j \in N),
$$

where $d(i)$ is the integral demand upper bound of agent $i \in N$.

\subsection{Lottery}

When designing a lottery to pick up a solution (a desired expected allocation), it is crucial to see that we have the integrality property of Conv(A) due to Corollary 4.2. Hence, given any (desired) expected allocation $\mathbf{E}\{\varphi\}$ in Conv(A), we need at most $|N| \times|E|$ (extreme) points in A that has positive probabilities of occurrence because of Carathéodory's theorem on convex polytopes in order to realize a lottery that gives the expected allocation $\mathrm{E}\{\varphi\}$.

Consequently, our problem becomes the following two:

(1) Find a point $\bar{\varphi}(=\mathbf{E}\{\varphi\})$ from among the polytope $\operatorname{Conv}(\mathbf{A})$ in an ordinally efficient and fair manner according to the preference profile $\mathcal{L}=\left(L^{i} \mid i \in N\right)$.

(2) Construct a lottery by finding a representation of $\bar{\varphi}$ as a convex combination of integral points of polytope Conv(A). The coefficients of the convex combination provide us with positive probabilities of a probability distribution over A that leads us to $\bar{\varphi}=\mathbf{E}\{\varphi\}$ in (19).

\footnotetext{
${ }^{6}$ We have assumed that the preference is strict and complete, i.e., $m=|E|$. This makes the arguments required in the sequel easier. When we allow indifference among types of goods, we can extend the result of Katta and Sethuraman [20] to our problem setting, which will be discussed elsewhere (see [14]).
}

, Vol. 1, No. 1, Article 1. Publication date: October 2017 
It heavily depends on the structure of the set $\mathrm{A}$ of feasible allocations whether we can find a desired expected solution $\bar{\varphi}$ and construct a lottery to realize $\bar{\varphi}$ in a computationally efficient way. Fortunately, it follows from Theorem 3.4 and Corollary 4.2 that $\mathrm{A}$ is the independent flow polytope and has a nice combinatorial structure as shown in the literature (see, e.g., [10]). We will see that the probabilistic serial (PS) mechanism by Bogomolnaia and Moulin [5] works surprisingly well for these general problem settings with submodular constraints.

\subsection{Brief historical remarks}

The problem considered here includes the following as special cases.

(a) The ordinary random assignment problem considered in the literature is mostly the case where $d=1 \in \mathbb{Z}_{>0}^{N}$ and $\mathbf{B}=\{1\} \subseteq \mathbb{Z}_{>0}^{E}$ (e.g., $[4,5,20]$ ). Here 1 denotes a vector of all ones of appropriate dimension (determined by the context).

(b) Kojima [21], Aziz [2], and Heo [18] considered a multi-unit demand case where $d \in \mathbb{Z}_{>0}^{N}$ and $\mathbf{B}=\{b\} \subseteq \mathbb{Z}_{>0}^{E}$ for some $b \in \mathbb{Z}_{>0}^{E}$.

Note that when $\mathbf{B}$ is a singleton set as in (a) and (b) above, the underlying (poly)matroid $(E, \rho)$ has the unique base and the rank function $\rho$ is modular.

\section{FINDING AN ORDINALLY EFFICIENT AND FAIR EXPECTED ALLOCATION}

We first show a procedure, Algorithm 1, which is an extension of the PS mechanism of Bogomolnaia and Moulin [5] and will then show that the computed point in $\operatorname{Conv}(A)$ is an ordinally efficient and fair expected allocation.

Let us define the base $x_{P}^{*} \in \mathrm{B}(\rho)$ associated with an allocation $P \in \operatorname{Conv}(\mathrm{A})$ by

$$
x_{P}^{*} \equiv \sum_{i \in N} P_{i} .
$$

Recall that for each $i \in N$ agent $i$ 's preference is given by (18), where $\left\{e_{1}^{i}, e_{2}^{i}, \cdots, e_{m}^{i}\right\}=E$ and $e_{1}^{i}$ is the most favorite (type of) good for agent $i$, and $\mathcal{L}$ is the profile of preferences $L^{i}(i \in N)$. Based on the collection (a multiset) of the first (most favorite) elements $e_{1}^{i}$ of all agents $i \in N$, define a nonnegative integral vector $b(\mathcal{L}) \in \mathbb{Z}_{\geq 0}^{E}$ by

$$
b(\mathcal{L})=\sum_{i \in N} d(i) \chi_{e_{1}^{i}}
$$

where we may have $e_{1}^{i}=e_{1}^{j}$ for distinct $i, j \in N$ and $d(i)$ is the integral demand upper bound of agent $i \in N$.

We also denote the random assignment problem by $\mathrm{RA}=\left(N, E, \mathcal{L}=\left(L^{i} \mid i \in N\right), d=(d(i) \mid i \in N),(E, \rho)\right)$.

During the execution of the following algorithm the current preference lists $L^{i}$ may get shorter because of removal of exhausted (or saturated) types of goods. Also note that $S_{p}$ is the set of types of goods saturated at stage $p$.

Algorithm 1: Extended PS Solution ${ }^{7}$

Input: A random assignment problem $\mathrm{RA}=(N, E, \mathcal{L}, d,(E, \rho))$.

Output: An expected allocation $P: N \times E \rightarrow \mathbb{R}_{\geq 0}$.

Step 0: For each $i \in N$ put $x^{i} \leftarrow 0 \in \mathbb{R}^{E}$ (the zero vector), and $x^{*} \leftarrow \mathbf{0} \in \mathbb{R}^{E}$.

Put $S_{0} \leftarrow \emptyset, p \leftarrow 1$, and $\lambda_{0} \leftarrow 0$.

Step 1: For current (updated) $\mathcal{L}=\left(L^{i} \mid i \in N\right)$, using $b(\mathcal{L})$ in (23), compute

$$
\lambda_{p}=\max \left\{t \geq \lambda_{p-1} \mid x^{*}+\left(t-\lambda_{p-1}\right) b(\mathcal{L}) \in \mathrm{P}(\rho)\right\} .
$$

\footnotetext{
${ }^{7}$ Katta and Sethuramann [20] used the term, extended PS, and we also adopt the same term, hoping no confusion.
} 
For each $i \in N$ put $x^{i} \leftarrow x^{i}+\left(\lambda_{p}-\lambda_{p-1}\right) d(i) \chi_{e_{1}^{i}}$.

Put $x^{*} \leftarrow x^{*}+\left(\lambda_{p}-\lambda_{p-1}\right) b(\mathcal{L})$ and $S_{p} \leftarrow \operatorname{sat}\left(x^{*}\right)$.

Step 2: Put $T_{p} \leftarrow S_{p} \backslash S_{p-1}$.

Update $L^{i}(i \in N)$ by removing all elements of $T_{p}$ from current $L^{i}(i \in N)$.

Step 3: If $\rho\left(S_{p}\right)<\rho(E)$, then put $p \leftarrow p+1$ and go to Step 1 .

Otherwise $\left(\rho\left(S_{p}\right)=\rho(E)\right)$ put $P(i, e) \leftarrow x^{i}(e)$ for all $i \in N$ and $e \in E$.

Return $P$.

As in [5], the parameter $t$ in (24) can be considered as time and each agent $i \in N$ eats the current top good $e_{1}^{i}$ at rate $d(i)$ per unit time.

\subsection{Examples}

To see the behavior of Algorithm 1 let us consider two illustrative examples given as follows.

Example I (A graphic matroidal constraint and unit demands): Let us consider a family $\mathcal{B}$ of feasible sets given by

$$
\mathcal{B}=\{X|X \subset E,| X \mid=2, X \neq\{a, b\}\} .
$$

This is a graphic matroid and $\mathcal{B}$ is the family of edge subsets of spanning trees of the graph shown as Figure 7 in Example 2.5.

Suppose that preferences of all agents are given as follows.

$$
\begin{array}{cc}
i \in N & \text { preference } L^{i} \\
1 & a>_{1} b>_{1} c>_{1} d \\
2 & a>_{2} c>_{2} b>_{2} d \\
3 & a>_{3} c>_{3} d>_{3} b \\
4 & b>_{4} a>_{4} d>_{4} c
\end{array}
$$

Also suppose that every agent has a unit demand, i.e., $d=1=(1,1,1,1) \in \mathbb{Z}^{N}$. Then by Algorithm 1 we get

$$
P=\begin{gathered}
a \\
1 \\
2 \\
3 \\
4
\end{gathered}\left(\begin{array}{cccc}
1 / 4 & 0 & 1 / 4 & 0 \\
1 / 4 & 0 & 1 / 4 & 0 \\
1 / 4 & 0 & 1 / 4 & 0 \\
0 & 1 / 4 & 0 & 1 / 4
\end{array}\right),
$$

where

and

$$
b(\mathcal{L})=\left(\begin{array}{cccc}
a & b & c & d \\
1+1+1, & 1, & 0, & 0
\end{array}\right), \quad S_{1}=\{a, b\}, \quad \lambda_{1}=1 / 4 \text { for } p=1
$$

$$
b(\mathcal{L})=(0,0,1+1+1,1), \quad S_{2}=\{a, b, c, d\}, \quad \lambda_{2}=\lambda_{1}+1 / 4 \text { for } p=2,
$$

and each row sum of $P$ is equal to $1 / 4+1 / 4=1 / 2$. Here, recall that $S_{p}$ is the set of saturated (or exhausted) goods after the $p$ th execution of Step 1 of Algorithm 1. Also, vectors $x_{\lambda_{p}}^{*}$ on $T_{p}=S_{p} \backslash S_{p-1}$ for $p=1,2$ are given by

$$
\begin{aligned}
& T_{1}=\{a, b\}, \quad T_{2}=\{c, d\}, \\
& x_{\lambda_{1}}^{*}(a)=3 / 4, \quad x_{\lambda_{1}}^{*}(b)=1 / 4, \quad x_{\lambda_{2}}^{*}(c)=3 / 4, \quad x_{\lambda_{2}}^{*}(d)=1 / 4 .
\end{aligned}
$$

Hence $x_{P}^{*}=(3 / 4,1 / 4,3 / 4,1 / 4)$. 
The following example has a polymatroidal constraint and multi-unit demands and supplies.

Example II: Consider $N=\{1,2,3,4\}$ and $E=\{a, b, c, d\}$ again. Let $(E, \rho)$ be a polymatroid with a rank function given by

$$
\rho(X)=\left\{\begin{array}{cl}
4|X| & \text { if }|X| \leq 2 \\
8 & \text { if }|X|>2
\end{array} \quad(\forall X \subseteq E)\right.
$$

Note that $(E, \rho)$ here is a symmetric polymatroid. Suppose that preferences of all agents are the same as Example I but $E=\{a, b, c, d\}$ should be regarded as a set of types of goods. Let a demand vector be given by $d=(4,2,1,1) \in$ $\mathbb{Z}^{N}$. Then by Algorithm 1 we have $P \in \mathbb{R}_{\geq 0}^{N \times E}$, as an $N \times E$ matrix, given as follows.

$$
P=\begin{array}{cccc}
a & b & c & d \\
1 \\
2 \\
3 \\
4
\end{array}\left(\begin{array}{cccc}
16 / 7 & 12 / 7 & 0 & 0 \\
8 / 7 & 0 & 6 / 7 & 0 \\
4 / 7 & 0 & 3 / 7 & 0 \\
0 & 4 / 7+3 / 7 & 0 & 0
\end{array}\right),
$$

where

$$
b(\mathcal{L})=(4+2+1, \quad 1, \quad 0, \quad 0), \quad S_{1}=\{a\}, \quad \lambda_{1}=4 / 7 \text { for } p=1
$$

and

$$
b(\mathcal{L})=(0,4+1,2+1,0), \quad S_{2}=\{a, b, c, d\}, \quad \lambda_{2}=\lambda_{1}+3 / 7 \text { for } p=2
$$

to get the expected allocation $P$ given above. Also, vectors $x_{\lambda_{p}}^{*}$, which are the restriction of $x_{P}^{*}$ on $T_{p}=S_{p} \backslash S_{p-1}$ for $p=1,2$, are given by

$$
\begin{aligned}
& T_{1}=\{a\}, \quad T_{2}=\{b, c, d\}, \\
& x_{\lambda_{1}}^{*}(a)=4, \quad x_{\lambda_{2}}^{*}(b)=19 / 7, \quad x_{\lambda_{2}}^{*}(c)=9 / 7, \quad x_{\lambda_{2}}^{*}(d)=0 .
\end{aligned}
$$

Hence $x_{P}^{*}=(4,19 / 7,9 / 7,0)$. Note that $\emptyset,\{a\},\{a, b, c\}$, and $\{a, b, c, d\}\left(=\operatorname{sat}\left(x_{P}^{*}\right)\right)$ are tight sets for $x_{P}^{*}$.

\subsection{Ordinal efficiency}

The following theorem can be shown in a very similar way as the corresponding one in [5]. However, it can be seen that the given proof heavily depends on the underlying submodularity structure, especially the one used for the arguments in [9].

Theorem 5.1. Algorithm 1 computes an expected allocation in $\operatorname{Conv}(\mathrm{A})$ that is ordinally efficient.

(Proof) By Algorithm 1 we get an expected allocation $P$ in $\operatorname{Conv}(\mathrm{A})$ together with a chain $S_{0}=\emptyset \subset S_{1} \subset \cdots \subset$ $S_{p}=E$. Let $Q$ be an arbitrary expected allocation in $\operatorname{Conv}(\mathrm{A})$ and suppose that $Q=P$ or $Q$ sd-dominates $P$. It suffices to prove $Q=P$.

At the $q$ th execution of Step 1 of Algorithm 1 define

$$
F_{q}=\left\{i \in N \mid e_{1}^{i} \in T_{q}\right\} .
$$

Let us denote $e_{1}^{i}$ (the top element in current $L^{i}$ ) at the $q$ th execution of Step 1 by $e_{1}^{i}(q)$ and suppose that for some integer $q^{*} \geq 1$ we have

$$
Q\left(i, e_{1}^{i}(q)\right)=P\left(i, e_{1}^{i}(q)\right) \quad\left(\forall q=1, \cdots, q^{*}-1, \forall i \in F_{q}\right)
$$

and we execute the $q^{*}$ th Step 1 . Then, because of Step 1 of Algorithm 1 we have

$$
\sum_{i \in F_{q}} P\left(i, e_{1}^{i}(q)\right)=\rho\left(S_{q}\right)-\rho\left(S_{q-1}\right) \quad\left(q=1, \cdots, q^{*}\right) .
$$


Since $Q=P$ or $Q$ sd-dominates $P$, it follows from (28) that $Q\left(i, e_{1}^{i}\left(q^{*}\right)\right) \geq P\left(i, e_{1}^{i}\left(q^{*}\right)\right)$ for all $i \in F_{q^{*}}$. Hence from (28) and (29) we must have

$$
Q\left(i, e_{1}^{i}\left(q^{*}\right)\right)=P\left(i, e_{1}^{i}\left(q^{*}\right)\right) \quad\left(\forall i \in F_{q^{*}}\right),
$$

since we have $\sum_{i \in F_{q^{*}}} Q\left(i, e_{1}^{i}\left(q^{*}\right)\right) \leq \rho\left(S_{q^{*}}\right)-\rho\left(S_{q^{*}-1}\right)$. Here note that

$$
\sum_{q=1}^{q^{*}} \sum_{i \in F_{q}} Q\left(i, e_{1}^{i}(q)\right) \leq \rho\left(S_{q^{*}}\right) .
$$

Now, note that when $q^{*}=1$, Equation (28) is void (and thus holds). Hence, by induction on $q=1, \cdots, p$, we have shown $Q=P$.

\subsection{Envy-freeness}

Recall the definition of normalized envy-freeness given in Section 4.2. We have the following theorem on normalized envy-freeness of the extended PS mechanism. The proof is actually a direct adaptation of the one given by Bogomolnaia and Moulin [5] and Schulman and Vazirani [28] for existing problem settings (also see [18]). It should be noted that by Algorithm 1 every agent $i \in N$ eats $d(i)$ units of goods per unit time, which is equivalent to considering $d(i)$ copies of every agent $i \in N$ such that each copy of agent $i$ has the same preference as agent $i$ but has a unit demand.

Theorem 5.2. Algorithm 1 computes an expected allocation $P$ that is normalized envy-free.

(Proof) It suffices to show that for any $i \in N$ and $k \in\{1, \cdots, m\}$ we have

$$
\frac{1}{d(i)} \sum_{\ell=1}^{k} P\left(i, e_{\ell}^{i}\right) \geq \frac{1}{d(j)} \sum_{\ell=1}^{k} P\left(j, e_{\ell}^{i}\right) \quad(\forall j \in N) .
$$

Define

$$
t_{k}^{i}=\frac{1}{d(i)} \sum_{\ell=1}^{k} P\left(i, e_{\ell}^{i}\right)
$$

When good $e_{k}^{i}$ is removed after an execution of Step 1 , all goods $e_{\ell}^{i}(\ell=1, \cdots, k)$ have been removed from $E$. It follows that for all $j \in N$ the time spent by agent $j$ to eat $e_{\ell}^{i}(\ell=1, \cdots, k)$ given by the sum of possible values $\frac{1}{d(j)} P\left(j, e_{\ell}^{i}\right)$ for goods $e_{\ell}^{i}(\ell=1, \cdots, k)$ is within $t_{k}^{i}$. Hence we must have

$$
t_{k}^{i} \geq \frac{1}{d(j)} \sum_{\ell=1}^{k} P\left(j, e_{\ell}^{i}\right) \quad(\forall j \in N) .
$$

\section{STRATEGY-PROOFNESS}

It is known that the extension of the PS mechanism of Bogomolnaia and Moulin to the case of multi-unit demands cannot be weakly strategy-proof in general $([3,6,18,21])$. Therefore, our polymatroidal extension is not weakly strategy-proof in general either. ${ }^{8}$

Note that a mechanism $M$ is weakly strategy-proof if for every input preference profile $\mathcal{L}$ the mechanism $M$ gives a solution (an expected allocation) $P$ such that for each agent $i \in N$ every misreport of agent $i$ 's preference

\footnotetext{
${ }^{8}$ Schulman and Vazirani [28] showed strategy-proofness of the PS mechanism under lexicographic preferences. It is left for future research to examine how their results can be adapted to our problem settings.
} 
results in a solution $Q$ satisfying that $Q_{i}$ does not sd-dominate $P_{i}$ for $i$. Here the strategy-proofness is concerned with the mechanism.

\subsection{Weak Nash equilibria}

Let us consider the concept of a weak Nash equilibrium, which is a property of the obtained solution. For a given input profile we say that the solution $P$ obtained by the mechanism $M$ is a weak Nash equilibrium if for each agent $i \in N$ every misreport of agent $i$ 's preference results in a solution $Q$ satisfying that $Q_{i}$ does not sd-dominate $P_{i}$ for $i$. That is, for a weak Nash equilibrium $P$ no agent $i$ can improve her expected allocation $P_{i}$ with respect to the sd-dominance relation by any misreport of her preference $L^{i}$.

We examine our polymatroidal extension and give a certain sufficient condition ${ }^{9}$ for our solution to be a weak Nash equilibrium. The result, Theorem 6.3, given below seems to be new even for the ordinary multi-unit demand case where the base polyhedron consists of a single base, i.e., $\mathrm{B}(\rho)=\{b\}$ for some $b \in \mathbb{Z}_{>0}^{E}$. In Section 6.2 we also prove the weak strategy-proofness in the special case of unit demands and matroidal supplies (shown in [13]).

6.1.1 Lemmas. We first prepare two lemmas to prove Theorem 6.3 concerned with a condition for our solution to be a weak Nash equilibrium.

Let us consider the 'eating process' (due to Bogomolnaia and Moulin [5]). By Algorithm 1 we have critical times

$$
\lambda_{0}=0<\lambda_{1}<\cdots<\lambda_{q}=\rho(E) / d(N)
$$

computed by (24), where $d(N)=\sum_{i \in N} d(i)$. At each critical time $\lambda_{k}>0, \mathcal{L}$ is updated by removing all the saturated types of goods from $\mathcal{L}$.

For each time $t$ with $\lambda_{k} \leq t \leq \lambda_{k+1}$ for $k \in\{0, \cdots, q-1\}$ we put

$$
x_{t}^{*}=x_{\lambda_{k}}^{*}+\left(t-\lambda_{k}\right) b\left(\mathcal{L}_{t}\right)
$$

where $\mathcal{L}_{t}=\left(L_{t}^{i} \mid i \in N\right)$ denotes the current $\mathcal{L}=\left(L^{i} \mid i \in N\right)$ at time $t$ and

$$
b\left(\mathcal{L}_{t}\right)=\sum_{i \in N} d(i) \chi_{e_{1}^{i}}
$$

with $e_{1}^{i}$ being the top element (type of good) of current $L_{t}^{i}$. We put $x_{0}^{*}=0$. Note that we have

$$
\operatorname{sat}\left(x_{t}^{*}\right)=\operatorname{sat}\left(x_{\lambda_{k}}^{*}\right) \quad\left(\forall t \in\left[\lambda_{k}, \lambda_{k+1}\right), \forall k \in\{0, \cdots, q-1\}\right) .
$$

Now, suppose that agent $1 \in N$ has a preference list $L^{1}$ and misreports her preference as $\bar{L}^{1}$. Put $\overline{\mathcal{L}}=\left(\bar{L}^{i} \mid i \in N\right)$ with $\bar{L}^{i}=L^{i}$ for $i \in N \backslash\{1\}$. For any original object $p$ (a parameter, a vector, etc.) defined under preference profile $\mathcal{L}$, let us denote by $\bar{p}$ the object $p$ defined under misreported preference profile $\overline{\mathcal{L}}$.

For each $e \in E$ define $N_{P}(e)=\{i \in N \mid P(i, e)>0\}$. For each $e \in E$ let $t(e)$ be the time when good $e$ is exhausted (or saturated). Also for each $e \in E$ and $i \in N_{P}(e)$ let $t_{0}^{i}(e)$ be the time when agent $i$ starts eating good $e$ (or the time when $e$ becomes the top element of current $L^{i}$ ).

LemMA 6.1. Let $L^{1}$ and $\bar{L}^{1}$ be given by

$$
\begin{aligned}
& L^{1}: w_{1}>\cdots>w_{s}>a>\cdots, \\
& \bar{L}^{1}: w_{1}>\cdots>w_{s}>z_{1}>\cdots>z_{s^{\prime}}>a>\cdots
\end{aligned}
$$

with $P(1, a)>0$ for some integers $s \geq 0$ and $s^{\prime} \geq 1$. Suppose that $\bar{t}(a)>t(a)$. Then, the following three statements hold during the execution of Algorithm 1 with current time $t<t(a)$.

(a) For each $i \in N \backslash\{1\}$ we have $e_{1}^{i} \geq_{i} \bar{e}_{1}^{i}$, where $e_{1}^{i}$ and $\bar{e}_{1}^{i}$ are the top elements of current $L_{t}^{i}$ for $\mathcal{L}_{t}$ and current $\bar{L}_{t}^{i}$ for $\overline{\mathcal{L}}_{t}$, respectively, and $\geq_{i}$ is the order of original $L^{i}$.

\footnotetext{
${ }^{9}$ The condition is very likely to hold for practical problems with a large number of agents.
} 
(b) For each $e \in E \backslash\left(\operatorname{sat}\left(\bar{x}_{t}^{*}\right) \cup\{a\}\right)$ we have $x_{t}^{*}(e) \leq \bar{x}_{t}^{*}(e)$.

(c) $\operatorname{sat}\left(x_{t}^{*}\right) \subseteq \operatorname{sat}\left(\bar{x}_{t}^{*}\right)$.

Moreover, we have

(d) $\bar{t}_{0}^{i}(a) \leq t_{0}^{i}(a) \quad\left(\forall i \in N_{P}(a) \backslash\{1\}\right)$.

(Proof) We can easily see from Algorithm 1 and the definition of $\mathcal{L}$ and $\overline{\mathcal{L}}$ that (a) implies

$$
b\left(\overline{\mathcal{L}}_{t}\right)(e) \geq b\left(\mathcal{L}_{t}\right)(e) \quad\left(\forall t \in[0, t(a)), \quad \forall e \in E \backslash\left(\operatorname{sat}\left(\bar{x}_{t}^{*}\right) \cup\{a\}\right)\right),
$$

which implies (b). Moreover, (c) follows from (b) and Lemma 3.3, where we restrict the ground set of polymatroid $(E, \rho)$ to $E \backslash\{a\}$ since $a$ is not saturated for both $x_{t}^{*}$ and $\bar{x}_{t}^{*}$ for $t<t(a)$ by the assumption. Also (d) easily follows from (a) (for all $t \in[0, t(a))$ ).

Hence it suffices to show that (a) holds for all $t \in[0, t(a))$, by induction on the indices $k$ of critical times $\lambda_{k}$ and $\bar{\lambda}_{k}$. First, note that (a) holds for $t \in\left[0, \min \left\{\lambda_{1}, \bar{\lambda}_{1}, t(a)\right\}\right)$ since $e_{1}^{i}=\bar{e}_{1}^{i}$ for all $i \in N \backslash\{1\}$.

We consider the following three cases (A), (B), and (C).

(A) Suppose that (a) holds for all $t \in\left[0, \lambda_{k}\right)$ for some $k \geq 1$ with $\lambda_{k}<t(a)$ and that $\lambda_{k}$ is not equal to any critical time for $\overline{\mathcal{L}}$, i.e., $\bar{\lambda}_{p}<\lambda_{k}<\bar{\lambda}_{p+1}$ for some $p$. Then it follows from Lemma 3.3 that at time $t=\lambda_{k}$, if $e_{1}^{i}$ for $i \in N \backslash\{1\}$ becomes saturated, then $e_{1}^{i}$ belongs to sat $\left(\bar{x}_{t}^{*}\right)$ and the new (non-saturated) $e_{1}^{i}$ satisfies $e_{1}^{i} \geq^{i} \bar{e}_{1}^{i}$. (Here we employ Lemma 3.3 by restricting the polymatroid to $E \backslash\{a\}$ since $a$ is not saturated for $x_{t}^{*}$ with $t<t(a)$.) Hence (a) holds for $t=\lambda_{k}$ and then so does for all $t \in\left[0, \min \left\{\lambda_{k+1}, \bar{\lambda}_{p+1}, t(a)\right\}\right)$.

(B) Suppose that (a) holds for all $t \in\left[0, \bar{\lambda}_{k}\right)$ for some $k \geq 1$ with $\bar{\lambda}_{k}<t(a)$ and that $\lambda_{p}<\bar{\lambda}_{k}<\lambda_{p+1}$ for some $p$. Then at time $t=\bar{\lambda}_{k}$ we have sat $\left(\bar{x}_{t}^{*}\right)$ enlarged and newly saturated $\bar{e}_{1}^{i}$ is replaced by the next non-saturated one in $\bar{L}_{t}^{i}$, current $\bar{L}^{i}$. Hence (a) holds for $t=\bar{\lambda}_{k}$ and then so does for all $t \in\left[0, \min \left\{\lambda_{p+1}, \bar{\lambda}_{k+1}, t(a)\right\}\right)$.

(C) Suppose that (a) holds for all $t \in\left[0, \lambda_{k}\right)$ for some $k \geq 1$ with $\lambda_{k}<t(a)$ and that $\lambda_{k}=\bar{\lambda}_{p}$ for some $p \geq 1$. Then at time $t=\bar{\lambda}_{p}\left(=\lambda_{k}\right)$ we have sat $\left(\bar{x}_{t}^{*}\right)$ enlarged and newly saturated $\bar{e}_{1}^{i}$ is replaced by the next non-saturated one in $\bar{L}_{t}^{i}$. Also, at time $t=\lambda_{k}\left(=\bar{\lambda}_{p}\right)$, if $e_{1}^{i}$ becomes saturated, then $e_{1}^{i}$ belongs to updated sat $\left(\bar{x}_{t}^{*}\right)$ and the new non-saturated $e_{1}^{i}$ satisfies $e_{1}^{i} \geq^{i} \bar{e}_{1}^{i}$ for possibly new non-saturated $\bar{e}_{1}^{i}$ (due to Lemma 3.3). Hence (a) holds for $t=\lambda_{k}\left(=\bar{\lambda}_{p}\right)$ and then so does for all $t \in\left[0, \min \left\{\lambda_{k+1}, \bar{\lambda}_{p+1}, t(a)\right\}\right)$.

This completes the proof of the present lemma by induction.

LEMMA 6.2. Under the same assumption as in Lemma 6.1, we have

$$
x_{t(a)}^{*}(a) \geq \bar{x}_{\bar{t}(a)}^{*}(a) .
$$

(Proof) Suppose that $\bar{t}(a)>t(a)$ and let $p$ and $q$ be integers such that $\bar{\lambda}_{p}<t(a) \leq \bar{\lambda}_{p+1}$ and $\lambda_{q}=t(a)$. It follows from Lemma 6.1 that for all $t \in\left[\bar{\lambda}_{p}, t(a)\right)$

$$
\operatorname{sat}\left(x_{t}^{*}\right) \subseteq \operatorname{sat}\left(\bar{x}_{t}^{*}\right), \quad x_{t}^{*}(e) \leq \bar{x}_{t}^{*}(e) \quad\left(\forall e \in E \backslash\left(\operatorname{sat}\left(\bar{x}_{t}^{*}\right) \cup\{a\}\right)\right) .
$$

Also we see from Lemma 6.1 and the continuity of $x_{t}^{*}$ in $t$ that at $t=t(a)$ we have

$$
x_{t(a)}^{*}(e) \leq \bar{x}_{t(a)}^{*}(e) \quad\left(\forall e \in E \backslash\left(\operatorname{sat}\left(\bar{x}_{t(a)}^{*}\right) \cup\{a\}\right)\right) .
$$

Define $\bar{y}_{\epsilon}=\bar{x}_{\bar{t}(a)-\epsilon}^{*}$ for any $\epsilon$ with $0<\epsilon \leq \bar{t}(a)$. Then, for a sufficiently small $\epsilon>0$ we have $a \notin \operatorname{sat}\left(\bar{y}_{\epsilon}\right)$ and

$$
\begin{array}{ll}
\bar{x}_{t(a)}^{*}(e)=\bar{y}_{\epsilon}(e) \quad\left(\forall e \in \operatorname{sat}\left(\bar{x}_{t(a)}^{*}\right)\right), \\
\bar{x}_{t(a)}^{*}(e) \leq \bar{y}_{\epsilon}(e) \quad\left(\forall e \in E \backslash \operatorname{sat}\left(\bar{x}_{t(a)}^{*}\right)\right) .
\end{array}
$$

Increase the values of $x_{t(a)}^{*}(e)$ for all $e \in \operatorname{sat}\left(\bar{y}_{\epsilon}\right)$ as much as possible while keeping the vector within $\mathrm{P}(\rho)$. Let $y^{*}$ be the resulting independent vector. Then we have

$$
\operatorname{sat}\left(y^{*}\right) \supseteq \operatorname{sat}\left(\bar{y}_{\epsilon}\right) \cup\{a\} .
$$


Put $A=\operatorname{sat}\left(y^{*}\right)$ and $B=\operatorname{sat}\left(\bar{y}_{\epsilon}\right)$. Then,

$$
x_{t(a)}^{*}(A)-x_{t(a)}^{*}(B)=y^{*}(A)-y^{*}(B) \geq \rho(A)-\rho(B)>\bar{y}_{\epsilon}(A)-\bar{y}_{\epsilon}(B),
$$

where the last inequality follows from the fact that $a \in A$ and $a \notin \operatorname{sat}\left(\bar{y}_{\epsilon}\right)$. Hence from (39)-(43) we have

$$
x_{t(a)}^{*}(a)>\bar{y}_{\epsilon}(a)=\bar{x}_{\bar{t}(a)-\epsilon}^{*}(a) \text {. }
$$

Since (44) holds for any sufficiently small $\epsilon>0$ and $\bar{x}_{t}^{*}$ is continuous in $t$, we have $x_{t(a)}^{*}(a) \geq \bar{x}_{\bar{t}(a)}^{*}(a)$.

6.1.2 Proof of weak Nash equilibrium. Suppose that we are given an expected allocation $P$ computed by Algorithm 1. Recall that $N_{P}(e)=\{i \in N \mid P(i, e)>0\}$ for all $e \in E$. The condition of the following theorem is very likely to be satisfied in practice. Note that the condition that $\left|N_{P}(e)\right| \neq 1$ for all $e \in E$ means that for every good, either more than one agent should compete for it or no agent at all.

THeOrem 6.3. Given the solution $P$ by Algorithm 1, if we have $\left|N_{P}(e)\right| \neq 1$ for all $e \in E$, then the solution $P$ is a weak Nash equilibrium.

(Proof) Suppose that $\left|N_{P}(e)\right| \neq 1$ for all $e \in E$. Recall that for each $e \in E, t(e)$ is the time when good $e$ is exhausted (or saturated). Also for each $e \in E$ and $i \in N_{P}(e), t_{0}^{i}(e)$ is the time when agent $i$ starts eating good $e$ (or the time when $e$ becomes the top element of current $L^{i}$ ).

For the solution $P$, if $P(1, a)=0$ for some $a \in E$, shifting good $a$ in $L^{1}$ toward the end of $L^{1}$ does not change the solution $P$. Hence we can assume

$(\dagger)$ goods $e$ with $P(1, e)>0$ appear consecutively in $L^{1}$ from the top of $L^{1}$.

Now suppose that for agent $1 \in N$ her preference is given by

$$
L^{1}: a>\cdots
$$

with $P(1, a)>0$ and she misreports her preference as

$$
\bar{L}^{1}: z_{1}>\cdots>z_{s}>a>\cdots
$$

with some integer $s \geq 1$. Let $\bar{P}$ be the PS solution obtained under the misreport. When $L^{1}$ is replaced by $\bar{L}^{1}$, we denote $t(e)$ and $t_{0}^{i}(e)$ by $\bar{t}(e)$ and $\bar{t}_{0}^{i}(e)$, respectively, for all $e \in E$ and $i \in N$, and also denote $x_{t}^{*}$ by $\bar{x}_{t}^{*}$.

Suppose that $\bar{P}_{1}$ sd-dominates $P_{1}$ or is equal to $P_{1}$ (i.e., $\left.\bar{P}_{1} \geq_{1}^{\text {sd }} P_{1}\right)$, where recall $\bar{P}_{1}=(\bar{P}(1, e) \mid e \in E)$ and $P_{1}=(P(1, e) \mid e \in E)$. Then it suffices to prove $\bar{P}_{1}=P_{1}$.

(I) Suppose that $\bar{t}(a)>t(a)$. Then from Lemma $6.1(\mathrm{~d})$ and Lemma 6.2 we have

$$
\begin{gathered}
\bar{t}_{0}^{i}(a) \leq t_{0}^{i}(a) \quad\left(\forall i \in N_{P}(a) \backslash\{1\}\right), \\
x_{t(a)}^{*}(a) \geq \bar{x}_{\bar{t}(a)}^{*}(a) .
\end{gathered}
$$

Now, since $\bar{t}(a)>t(a)$ and $N_{P}(a) \backslash\{1\} \neq \emptyset$ by the assumption, it follows from (47) and (48) that $\bar{P}(1, a)<P(1, a)$, a contradiction. Hence we have $\bar{t}(a) \leq t(a)$ and

$$
\bar{P}(1, a)=\bar{t}(a)-\left(\bar{P}\left(1, z_{1}\right)+\cdots+\bar{P}\left(1, z_{s}\right)\right) \leq t(a)=P(1, a) .
$$

Since from the assumption that $\bar{P}_{1} \geq_{1}^{\text {sd }} P_{1}$ we must have $\bar{P}(1, a) \geq P(1, a)$, it follows from (49) that

$$
\bar{P}(1, a)=P(1, a), \quad \bar{P}\left(1, z_{1}\right)=\cdots=\bar{P}\left(1, z_{s}\right)=0 .
$$

The latter relation in (50) implies

- elements $z_{1}, \cdots, z_{s}$ are saturated at time $t=0$ for $\overline{\mathcal{L}}$. 
Since shifting elements $z_{1}, \cdots, z_{s}$ toward the end of $\bar{L}^{1}$ does not change $\bar{P}$, it suffices to consider that $L^{1}$ and $\bar{L}^{1}$ are given as

$$
\begin{aligned}
& L^{1}: a>b>\cdots, \\
& \bar{L}^{1}: a>z_{1}^{\prime}>\cdots>\cdots>z_{s^{\prime}}^{\prime}>b>\cdots
\end{aligned}
$$

for some $\left\{z_{1}^{\prime}, \cdots, z_{s^{\prime}}^{\prime}\right\} \subseteq E \backslash\{a, b\}$ with an integer $s^{\prime} \geq 0$.

(II) If $P(1, b)=0$, then it easily follows from the assumption ( $\dagger$ ) that $P_{1}=\bar{P}_{1}$. Hence suppose $P(1, b)>0$. Then by the same arguments as in (I), using Lemma 6.2 again, we can show

(1) $\bar{t}(b) \leq t(b)$,

(2) $\bar{P}(1, b)=P(1, b), \quad \bar{P}\left(1, z_{1}^{\prime}\right)=\cdots=\bar{P}\left(1, z_{s^{\prime}}^{\prime}\right)=0$,

(3) elements $z_{1}^{\prime}, \cdots, z_{s^{\prime}}^{\prime}$ are saturated at time $t=\bar{t}(a)(=t(a))$ for $\overline{\mathcal{L}}$

and it suffices to consider the case where there is no element between $a$ and $b$ in $\bar{L}^{1}$.

(III) Further repeating this argument, we can show that $\bar{P}_{1}=P_{1}$.

Theorem 6.3 is rephrased as follows. (Note that matrix $P \in \mathbb{R}^{N \times E}$ has the row set $N$ and the column set $E$.)

- If no column of $P$ contains exactly one non-zero entry, the extended PS solution $P$ computed by Algorithm 1 is a weak Nash equilibrium.

Theorem 6.3 has very useful practical implications from the point of view of strategy-proofness. The condition that $\left|N_{P}(e)\right| \neq 1(\forall e \in E)$ is very likely to be satisfied when the number $|N|$ of 'agents' is significantly large, compared with the number $|E|$ of 'types of goods' such as the assignment of students to courses.

\subsection{Weak strategy-proofness in case of unit demands and matroidal supplies}

We show that when the polymatroid $(E, \rho)$ is a matroid and agents have unit demands, the extended PS mechanism (Algorithm 1 ) is weakly strategy-proof, where the matroidal $\{0,1\}$ property plays a crucial role.

Consider the random assignment problem $\mathrm{RA}=\left(N, E, \mathcal{L}=\left(L^{i} \mid i \in N\right), d,(E, \rho)\right)$ and suppose that the underlying polymatroid $(E, \rho)$ is a matroid and agents have unit demands, i.e., $d=1$. We assume that $\rho(E)=|N|$.

Lemma 6.4. Suppose that $L^{1}$ and $\bar{L}^{1}$ are given by (35) and (36) and that $P(1, a)>0$. Suppose that $\bar{t}(a)>t(a)$. Then we have $\bar{P}(1, a) \leq P(1, a)$. Moreover, we have $\bar{P}(1, a)=P(1, a)(>0)$ only when $\bar{P}\left(1, z_{1}\right)=\cdots=\bar{P}\left(1, z_{s^{\prime}}\right)=0$.

(Proof) Because of Theorem 6.3 it suffices to consider the case where $\left|N_{P}(a)\right|=1$. Suppose that $L^{1}$ and $\bar{L}^{1}$ are given by (35) and (36) and that $P(1, a)>0$.

Suppose $\left|N_{P}(a)\right|=1$, i.e., $N_{P}(a)=\{a\}$. From Lemma 6.2 we have

$$
P(1, a)=x_{t(a)}^{*}(a) \geq \bar{x}_{\bar{t}(a)}^{*}(a) \geq \bar{P}(1, a) .
$$

If $\left|\bar{N}_{\bar{P}}(a)\right| \geq 2$, then the last inequality in (53) should hold with strict inequality. Hence it suffices to consider the case where $\left|\bar{N}_{\bar{P}}(a)\right|=1=\left|N_{P}(a)\right|$. Moreover, since $\bar{t}(a)>t(a)$ by the assumption, it follows from (53) that

$$
\bar{P}\left(1, z_{1}\right)+\cdots+\bar{P}\left(1, z_{s^{\prime}}\right)>0 .
$$

We show that this leads us to $\bar{P}(1, a)<P(1, a)$.

Increase the values of $x_{t(a)}^{*}(e)$ for all $e \in \operatorname{sat}\left(\bar{x}_{t(a)}^{*}\right)$ as much as possible while keeping the vector within $\mathrm{P}(\rho)$. (Here note that $a \notin \operatorname{sat}\left(\bar{x}_{t(a)}^{*}\right)$ since $\bar{t}(a)>t(a)$.) Let $z^{*}$ be the resulting independent vector. Then, since $X \equiv \operatorname{sat}\left(\bar{x}_{t(a)}^{*}\right)$ and $Z \equiv \operatorname{dep}\left(x_{t(a)}^{*}, a\right)$ are tight for $z^{*}$, we have

$$
z^{*}(X \cup Z)=\rho(X \cup Z) .
$$

Consider the following two cases (i) and (ii). 
Case (i): $(Z \backslash\{a\}) \backslash X \neq \emptyset$. In this case, it follows from (39) that

$$
\bar{x}_{\bar{t}(a)}^{*}(e)>\bar{x}_{t(a)}^{*}(e) \geq x_{t(a)}^{*}(e) \quad(\forall e \in(Z \backslash\{a\}) \backslash X) .
$$

(Here note that for all $e \in(Z \backslash\{a\}) \backslash X$ we have $\bar{x}_{t(a)}^{*}(e) \geq x_{t(a)}^{*}(e)>0$, where we have $x_{t(a)}^{*}(e)>0$ because of the definition of $Z=\operatorname{dep}\left(x_{t(a)}^{*}, a\right)$, and hence $e$ is the top element of current $L_{t}^{i}$ of at least one agent $i \in N \backslash\{1\}$ for $\overline{\mathcal{L}}_{t}$ (as well as for $\mathcal{L}_{t}$ ) at time $t=t(a)$.) Hence, if $\bar{x}_{\bar{t}(a)}^{*}(a)=x_{t(a)}^{*}(a)$, then from (55) and (56) we have

$$
\bar{x}_{\bar{t}(a)}^{*}(X \cup Z)>z^{*}(X \cup Z)=\rho(X \cup Z),
$$

a contradiction. We thus have $\bar{P}(1, a)=\bar{x}_{\bar{t}(a)}^{*}(a)<x_{t(a)}^{*}(a)=P(1, a)$.

Case (ii): $(Z \backslash\{a\}) \backslash X=\emptyset$. In this case, it follows from (55) that

$$
P(1, a)=x_{t(a)}^{*}(a)=z^{*}(X \cup\{a\})-z^{*}(X)=\rho(X \cup\{a\})-\rho(X)=1,
$$

where note that $X \cup Z=X \cup\{a\}$ and $P(1, a)>0$. It follows from (54) that $\bar{P}(1, a)=\bar{x}_{\bar{t}(a)}^{*}(a)<1=P(1, a)$.

It should be noted that the above proof in Case (ii) depends on the matroidal $\{0,1\}$ property.

THEOREM 6.5. When the underlying polymatroid $(E, \rho)$ is a matroid and agents have unit demands, the extended PS mechanism given by Algorithm 1 is weakly strategy-proof.

(Proof) The present theorem can be shown similarly as Theorem 6.3, based on Lemma 6.4.

\section{DESIGNING A LOTTERY}

Now we examine how to compute an expression of the solution $P$, obtained by Algorithm 1, as a convex combination of integral (possibly extreme) points $Q^{(k)}(k \in K)$ of $\operatorname{Conv}(\mathbf{A})$ as follows.

$$
P=\sum_{k \in K} v_{k} Q^{(k)}
$$

where $v_{k}>0$ for all $k \in K$ and $\sum_{k \in K} v_{k}=1$.

We will show that we can always compute a required convex combination representation (59) in an efficient way (see Algorithm 2 given in Section 7.2). With the aid of polymatroidal results achieved in $[8-10,16,24]$ we can construct a lottery to attain $P$ by finding the expression as in (59).

\subsection{Computing the probability distribution}

The proposed Algorithm 2 for efficiently computing an expression (59) is basically a standard procedure to obtain an expression of a given point in a polytope $P^{*}$ by a convex combination of its extreme points ${ }^{10}$, but it is crucial how efficiently we can compute an end point of the intersection of a line and a base polytope $([16,24])$ and can identify the unique minimal face of $P^{*}$ containing any given point in $P^{*}([10])$.

Put $P^{*}=\operatorname{Conv}(\mathrm{A})$. For the expected allocation matrix $P$ (or independent flow $\varphi_{P}$ ) and base $x_{P}^{*} \in \mathrm{B}(\rho)$ computed by Algorithm 1 we first consider the unique minimal face of $P^{*}$ containing $\varphi_{P}$.

\footnotetext{
${ }^{10}$ This is an adaptation of a standard procedure for finding an expression of a given point $x$ in a relative interior of a polytope $P$ as a convex combination of extreme points of $P$. First find an extreme point $y$ and extend a half-line from $y$ through $x$ as far as we reach a boundary point $z$ in a minimal face $F$ (containing $z$ ) of $P$. Since $x$ is expressed as a convex combination of $y$ and $z$, a desired convex combination expression can be obtained if $z$ is expressed as a convex combination of extreme points of face $F$, a polytope of dimension smaller than $P$. Here note that $z$ is within the relative interior of face $F$ because of the minimality of face $F$. We thus have a required algorithm.
} 
Denote by $\mathcal{D}\left(x_{P}^{*}\right)$ the set of all tight sets for $x_{P}^{*}$ in $\mathrm{B}(\rho)$, where $\mathcal{D}\left(x_{P}^{*}\right)$ is closed with respect to the binary operations of set union and intersection and is a distributive lattice, due to Lemma 3.1 (also see [10]). Let a maximal chain of $\mathcal{D}\left(x_{P}^{*}\right)$ be given by

$$
\hat{C}: \hat{S}_{0}=\emptyset \subset \cdots \subset \hat{S}_{p}=E .
$$

The chain of tight sets obtained during the execution of Algorithm 1 is a subchain of (60). A maximal chain $\hat{C}$ is determined by the dependence structure associated with $\operatorname{dep}\left(x_{P}^{*}, e\right)$ for all $e \in E$ and can be computed in strongly polynomial time ([10]).

For each $q=1, \cdots, p$ consider the minor, denoted by $\mathbf{P}_{q}$, of polymatroid $(E, \rho)$ obtained by its restriction to $\hat{S}_{q}$ followed by the contraction of $\hat{S}_{q-1}$. The minor $\mathbf{P}_{q}$ is the polymatroid on $\hat{T}_{q} \equiv \hat{S}_{q} \backslash \hat{S}_{q-1}$ with the rank function $\rho_{q}$ given by

$$
\rho_{q}(X)=\rho\left(X \cup \hat{S}_{q-1}\right)-\rho\left(\hat{S}_{q-1}\right) \quad\left(\forall X \subseteq \hat{T}_{q}\right) .
$$

Also denote by $x_{q}^{*}$ the restriction of $x_{P}^{*}$ to $\hat{T}_{q}\left(=\hat{S}_{q} \backslash \hat{S}_{q-1}\right)$. Then $x_{q}^{*}$ is a base of the polymatroid $\left(\hat{T}_{q}, \rho_{q}\right)$, i.e., $x_{q}^{*} \in \mathrm{B}\left(\rho_{q}\right)$. Note that $x_{P}^{*}$ is a base of the direct sum $\oplus_{q=1}^{p} \mathbf{P}_{q}$ of minors $\mathbf{P}_{q}(q=1, \cdots, p)$. Let $\hat{\rho}$ be the rank function of polymatroid $\oplus_{q=1}^{p} \mathbf{P}_{q}$. It should be noted that because of the maximality of chain $\hat{C}$, for each $q=1, \cdots, p$ the base polyhedron $\mathrm{B}\left(\rho_{q}\right) \subseteq \mathbb{R}^{\hat{T}_{q}}$ is of dimension $\left|\hat{T}_{q}\right|-1$ and base $x_{q}^{*}$ is within the relative interior of $\mathrm{B}\left(\rho_{q}\right)$ and that $x_{P}^{*}$ is within the relative interior of the base polyhedron $\mathrm{B}(\hat{\rho})$ of $\oplus_{q=1}^{p} \mathrm{P}_{q}$, which is the unique minimal face of $\mathrm{B}(\rho)$ containing $x_{P}^{*}$. (See $[10$, Chapter II].)

Put

$$
\begin{aligned}
& \hat{A}^{0}=\left\{a \in A \mid \varphi_{P}(a)=0\right\}, \\
& \hat{A}^{+}=A \backslash \hat{A}^{0}, \\
& \hat{I}=\left\{i \in N \mid \partial^{+} \varphi_{P}(i)=d(i)\right\} .
\end{aligned}
$$

Then, define a face of $P^{*}$ containing $\varphi_{P}$ by

$$
P^{*}\left(\varphi_{P}\right)=\left\{\varphi \in P^{*} \mid \forall i \in \hat{I}: \partial^{+} \varphi(i)=d(i), \forall a \in \hat{A}^{0}: \varphi(a)=0, \partial^{-} \varphi \in \mathrm{B}(\hat{\rho})\right\} .
$$

We can show the following lemma.

Lemma 7.1. The polytope $P^{*}\left(\varphi_{P}\right)$ is the unique minimal face of $P^{*}$ containing $\varphi_{P}$.

(Proof) In the system of inequalities (and equations) that defines $P^{*}$, the given $\varphi_{P}$ satisfies $\partial^{+} \varphi_{P}(i)=d(i)$ for all $i \in \hat{I}, \varphi_{P}(a)=0$ for all $a \in \hat{A}^{0}$, and

$$
\partial^{-} \varphi_{P}(X)=\rho(X) \quad\left(\forall X \in \mathcal{D}\left(x_{P}^{*}\right)\right),
$$

which includes all the inequalities for $P^{*}$ satisfied with equality by $\varphi_{P}$. Note that (66) is implied by

$$
\partial^{-} \varphi_{P}(X)=\rho(X) \quad\left(\forall X \in C\left(x_{P}^{*}\right)\right),
$$

since $\mathcal{D}\left(x_{P}^{*}\right)$ is a distributive lattice and $\rho$ is modular on $\mathcal{D}\left(x_{P}^{*}\right)$. Also note that the system of equations (67) together with $\partial^{-} \varphi_{P} \in \mathrm{B}(\rho)$ is equivalent to $\partial^{-} \varphi_{P} \in \mathrm{B}(\hat{\rho})$. Hence (65) defines the unique minimal face of $P^{*}$ containing $\varphi_{P}$.

We can easily see that polytope $P^{*}\left(\varphi_{P}\right)$ given by (65) is the set of feasible flows of value $\rho(E)$ in another independent flow network which we denote by $\hat{\mathcal{N}}_{\varphi_{P}}$. Here $\hat{\mathcal{N}}_{\varphi_{P}}$ consists of the bipartite graph $G=\left(S^{+}, S^{-} ; A\right)$ with $S^{+}=N, S^{-}=E$, and $A=N \times E$, a capacity function $\hat{c}: A \rightarrow \mathbb{R}_{\geq 0}$ with $\hat{c}(a)=0\left(a \in \hat{A}^{0}\right)$ and $\hat{c}(a)=c(a)$ $\left(a \in \hat{A}^{+}\right)$, a polymatroid $(E, \hat{\rho})$ on $S^{-}=E$, and a polymatroid on $S^{+}=N$ given by the direct sum of polymatroids $\left(\hat{I}, \tilde{\rho}_{1}\right)$ and $\left(N \backslash \hat{I}, \tilde{\rho}_{2}\right)$ with $\tilde{\rho}_{1}(X)=d(X)$ for $X \subseteq \hat{I}$ and $\tilde{\rho}_{2}(X)=\min \{d(X), \rho(E)-d(\hat{I})\}$ for $X \subseteq N \backslash \hat{I}$, where $\hat{A}^{0}$, $\hat{A}^{+}$, and $\hat{I}$ are defined by (62)-(64). 


\subsection{Randomized mechanism}

We begin with base $x_{1}^{*} \equiv x_{P}^{*} \in \mathrm{B}(\hat{\rho})$ and independent flow $\hat{\varphi}_{1} \equiv \varphi_{P}$ in network $\hat{\mathcal{N}}_{\varphi_{P}}$ such that $x_{1}^{*}=\partial^{-} \hat{\varphi}_{1}$. If $\varphi_{P}$ is already integer-valued, we are done. Hence we assume that $\varphi_{P}$ is not integer-valued. Perform the following procedure to compute an expression (59). Here recall that an $N \times E$ matrix $P$ and a flow $\varphi_{P}: N \times E \rightarrow \mathbb{R}$ are identified with each other.

Algorithm 2: Convex Combination

Input: $\mathrm{A}$ base $x_{1}^{*} \equiv x_{P}^{*} \in \mathrm{B}(\hat{\rho})$ and a (non-integral) independent flow $\hat{\varphi}_{1} \equiv \varphi_{P}$ in $\hat{\mathcal{N}}_{\varphi_{P}}$.

Output: Integer-valued feasible allocations $\varphi_{s}$ for all $s=1, \cdots, t+1$ and coefficients $\beta_{s}^{*}$ for all $s=1, \cdots, t$.

Step 1: Put $t \leftarrow 1$.

Step 2: Find an integer-valued independent flow $\varphi_{t}$ in $\hat{\mathcal{N}}_{\hat{\varphi}_{t}}$.

Step 3: Compute

$$
\beta_{t}^{*}=\max \left\{\beta>0 \mid \hat{\varphi}_{t}+\beta\left(\hat{\varphi}_{t}-\varphi_{t}\right) \in P^{*}\left(\hat{\varphi}_{t}\right)\right\}
$$

Step 4: Put $\hat{\varphi}_{t+1} \leftarrow \hat{\varphi}_{t}+\beta_{t}^{*}\left(\hat{\varphi}_{t}-\varphi_{t}\right)$ and $x_{t+1}^{*} \leftarrow x_{t}^{*}+\beta_{t}^{*}\left(x_{t}^{*}-\partial^{-} \varphi_{t}\right)$.

Step 5: If flow $\hat{\varphi}_{t+1}$ is not integer-valued, then put $t \leftarrow t+1$ and go to Step 2 .

Otherwise put $\varphi_{t+1} \leftarrow \hat{\varphi}_{t+1}$.

Return $\varphi_{s}$ for all $s=1, \cdots, t+1$ and $\beta_{s}^{*}$ for all $s=1, \cdots, t$.

During the execution of the above procedure, $P^{*}\left(\hat{\varphi}_{t}\right)$ appearing in (68) is the unique minimal face of $P^{*}$ containing $\hat{\varphi}_{t}$, due to Lemma 7.1. At the $t$ th execution of Step 3 with current rank function $\hat{\rho}$ we have the unique minimal face $\mathrm{B}(\hat{\rho})$ of $\mathrm{B}(\rho)$ containing $x_{t}^{*}$. Then $\beta_{t}^{*}$ in $(68)$ is the maximum value of $\beta$ that satisfies

$$
\begin{aligned}
& \partial^{+} \hat{\varphi}_{t}(i) \leq d(i) \quad(\forall i \in N), \\
& \hat{\varphi}_{t}(a)+\beta\left(\hat{\varphi}_{t}(a)-\varphi_{t}(a)\right) \geq 0 \quad\left(\forall a \in \hat{A}_{t}^{+}\right), \\
& x_{t}^{*}+\beta\left(x_{t}^{*}-\partial^{-} \varphi_{t}\right) \in \mathrm{B}(\hat{\rho}),
\end{aligned}
$$

where $\hat{A}_{t}^{+}=\left\{a \in A \mid \hat{\varphi}_{t}(a)>0\right\}$. Note that since $\hat{\varphi}_{t}$ is within the relative interior of $P^{*}\left(\hat{\varphi}_{t}\right)$, we get $\beta_{t}^{*}>0$. We can compute $\beta_{t}^{*}$ in strongly polynomial time (due to Nagano [24] and Goemans et al. [16]) by using any strongly polynomial submodular function minimization algorithm. Also note that the final value of $t$ is $\mathrm{O}(|N||E|)$ since every execution of Step 3 and Step 4 makes at least one strict inequality in (69) or (70) hold with equality or makes the length of a maximal chain $C\left(x_{t+1}^{*}\right)$ greater than that of $C\left(x_{t}^{*}\right)$.

From the output $\varphi_{s}$ for all $s=1, \cdots, t+1$ and $\beta_{s}^{*}$ for all $s=1, \cdots, t$ we have

$$
\hat{\varphi}_{s+1}=\left(1+\beta_{s}^{*}\right) \hat{\varphi}_{s}-\beta_{s}^{*} \varphi_{s} \quad(\forall s=1, \cdots t),
$$

or

$$
\hat{\varphi}_{s}=\left(1+\beta_{s}^{*}\right)^{-1}\left(\hat{\varphi}_{s+1}+\beta_{s}^{*} \varphi_{s}\right) \quad(\forall s=1, \cdots t) .
$$

Eliminating $\hat{\varphi}_{s}$ for $s=1, \cdots, t$ and using $\hat{\varphi}_{t+1}=\varphi_{t+1}$, we can obtain the following expression.

$$
\varphi_{P}\left(=\hat{\varphi}_{1}\right)=\sum_{s=1}^{t+1} v_{s} \varphi_{s}
$$

for some convex combination coefficients $v_{s}(s=1, \cdots, t+1)$. Each integer-valued flow $\varphi_{s}$ gives a desired assignment matrix $Q^{(s)}$, and $v_{s}(s=1, \cdots, t+1)$ the desired probability distribution on the set of assignment 
matrices $Q^{(s)}(s=1, \cdots, t+1)$. Note that (74) is equivalent to

$$
P=\sum_{s=1}^{t+1} v_{s} Q^{(s)},
$$

which thus can be computed in strongly polynomial time.

Now we have the following procedure.

Randomized_Mechanism

Step 1: Compute $P$ by using Algorithm 1.

Step 2: Compute an expression (59) by using Algorithm 2.

Step 3: Choose an allocation from among $Q^{(k)}(k \in K)$ by the lottery with the probability distribution $v_{k}(k \in K)$.

Consequently, we have shown the following theorem.

Theorem 7.2. By using Algorithm 1 to find the expected allocation matrix $P$ and using Algorithm 2 to construct a lottery realizing $P$, Randomized_Mechanism generates a feasible integral allocation in strongly polynomial time whose expectation is equal to the desired solution $P$.

It should be noted that defining $\underline{u}=\left\lfloor\partial^{-} \varphi_{P}\right\rfloor$ and $\bar{u}=\left\lceil\partial^{-} \varphi_{P}\right\rceil$, we may replace the original base polyhedron $\mathrm{B}(\rho)$ by its vector minor (the restriction by $\bar{u}$ and the contraction by $\underline{u}$ )

$$
\mathrm{B}(\rho)_{\underline{u}}^{\bar{u}}=\{x \in \mathrm{B}(\rho) \mid \underline{u} \leq x \leq \bar{u}\},
$$

where for any real $z\lfloor z\rfloor$ and $\lceil z\rceil$ are, respectively, the integer $z^{*}$ nearest to $z$ satisfying $z^{*} \leq z$ and $z \leq z^{*}$, and for any $x \in \mathbb{R}^{E}\lfloor x\rfloor=(\lfloor x(e)\rfloor \mid e \in E)$ and $\lceil x\rceil=(\lceil x(e)\rceil \mid e \in E)$. Also we define lower and upper capacities $\underline{c}$ and $\bar{c}$ on $\operatorname{arcs}$ in $A$ as $\underline{c}(a)=\left\lfloor\varphi_{P}(a)\right\rfloor$ and $\bar{c}(a)=\left\lceil\varphi_{P}(a)\right\rceil$ for all $a \in A$, and consider the independent flows with these capacities and base polyhedron $\mathrm{B}(\rho)_{\underline{u}}^{\bar{u}}$. Then, we can adapt the procedure, Algorithm 2 , to the independent flow network modified above and the obtained $Q^{(k)}(k \in K)$ become closer to $P$ than those obtained for the original network $\mathcal{N}$. This may give a favorable lottery in practice, especially for a polymatroid $(E, \rho)$ with large $\rho(E)$ or $d(N)$.

\section{CONCLUDING REMARKS}

We have considered the random assignment problem with submodular constraints on goods and have shown the following:

(1) Theorems 5.1 and 5.2 that the probabilistic serial (PS) mechanism of Bogomolnaia and Moulin [5] can naturally be extended to give an ordinally efficient and normalized envy-free solution for polymatroidal constraints.

(2) A sufficient condition (Theorem 6.3) that guarantees that the computed PS solution is a weak Nash equilibrium, which is very likely to be satisfied for practical problems with a large number of agents.

(3) Weak strategy-proofness (Theorem 6.5) of the extended PS mechanism in case of unit demands and matroidal supplies.

In our earlier manuscript [12] we investigated the random assignment problem with matroidal constraints in more details, where we examined a characterization of the extended PS solution by min-cost independent flows and by lexicographic optimality, which we have omitted here. 


\section{ACKNOWLEDGMENTS}

We are very grateful to the three anonymous reviewers for their useful comments that improved the presentation of the present paper.

\section{REFERENCES}

[1] A. Abdulkadíroğlu and T. Sönmez, 1998: Random serial dictatorship and the core from random endowments in house allocation problems. Econometrica 66, 3 (May 1998) 689-701.

[2] H. Aziz, 2015: Random assignment with multi-unit demands. arXiv:1401.7700v3 [cs.GT] 19 Jun 2015.

[3] A. Bogomolnaia, 2015: Random assignment: redefining the serial rule. Fournal of Economic Theory 158, A (July 2015) 308-318.

[4] A. Bogomolnaia and E. J. Heo, 2012: Probabilistic assignment of objects: characterizing the serial rule. fournal of Economic Theory 147, 5 (Sept. 2012) 2072-2082.

[5] A. Bogomolnaia and H. Moulin, 2001: A new solution to the random assignment problem. fournal of Economic Theory 100, 2 (Oct. 2001) $295-328$.

[6] E. Budish, Y.-K. Che, F. Kojima, and P. Milgrom, 2013: Designing random allocation mechanisms: Theory and applications. American Economic Review 103, 2 (April 2013) 585-623.

[7] J. Edmonds, 1970: Submodular functions, matroids, and certain polyhedra. In Proceedings of the Calgary International Conference on Combinatorial Structures and Their Applications (R. Guy, H. Hanani, N. Sauer and J. Schönheim, eds., Gordon and Breach, New York, 1970) 69-87.

[8] S. Fujishige, 1978: Algorithms for solving the independent-flow problems. Fournal of the Operations Research Society of Japan 21, 2 (June 1978) 189-204

[9] S. Fujishige, 1980: Lexicographically optimal base of a polymatroid with respect to a weight vector. Mathematics of Operations Research 2, 2 (May 1980) 186-196.

[10] S. Fujishige, 2005: Submodular Functions and Optimization (Second Edition). Elsevier, Amsterdam.

[11] S. Fujishige, 2009: Theory of principal partitions revisited. In: W. Cook, L. Lovász, and J. Vygen (Editors): Research Trends in Combinatorial Optimization (Springer, Berlin, 2009) 127-162.

[12] S. Fujishige, Y. Sano, and P. Zhan, 2016: A solution to the random assignment problem with a matroidal family of goods. RIMS Preprint RIMS-1852, Kyoto University, May 2016.

[13] S. Fujishige, Y. Sano, and P. Zhan, 2016: An extended probabilistic serial mechanism to the random assignment problem with multi-unit demands and polymatroidal supplies. RIMS Preprint RIMS-1866, Kyoto University, November 2016.

[14] S. Fujishige, Y. Sano, and P. Zhan, 2017: Submodular Optimization Views on the Random Assignment Problem. RIMS Preprint RIMS-1881, Kyoto University, September 2017.

[15] G. Gallo, M. D. Grigoriadis, and R. E. Tarjan, 1989: A fast parametric maximum flow algorithm and applications. SIAM fournal on Computing 18, 1 (Feb. 1989) 30-55.

[16] M. X. Goemans, S. Gupta, and P. Jaillet, 2017: Discrete Newton's algorithm for parametric submodular function minimization. In Proceedings of IPCO 2017 (F. Eisenbrand and J. Koenemann (Eds.): IPCO 2017, LNCS 10328) 212-227.

[17] T. Hashimoto, D. Hirata, O. Kesten, M. Kurino, and M. U. Ünver, 2014: Two axiomatic approaches to the probabilistic serial mechanism. Theoretical Economics 9, 1 (Jan. 2014) 253-277.

[18] E. J. Heo, 2014: Probabilistic assignment problem with multi-unit demands: a generalization of the serial rule and its characterization. fournal of Mathematical Economics 54 (Oct. 2014) 40-47.

[19] E. J. Heo and V. Manjunath, 2015: Implementation in stochastic dominance Nash equilibria. Social Choice and Welfare 48, 1 (Jan. 2017) 5-30.

[20] A.-K. Katta and J. Sethuraman, 2006: A solution to the random assignment problem on the full preference domain. Fournal of Economic Theory 131, 1 (Nov. 2006) 231-250.

[21] F. Kojima, 2009: Random assignment of multiple indivisible objects. Mathematical Social Sciences 57, 1 (Jan. 2009) 134-142.

[22] F. Kojima and M. Manea, 2010: Incentives in the probabilistic serial mechanism. Fournal of Economic Theory 145, 1 (Jan. 2010) 106-123.

[23] N. Megiddo, 1974: Optimal flows in networks with multiple sources and sinks. Mathematical Programming 7, 1 (Dec. 1974) 97-107.

[24] K. Nagano, 2007: A strongly polynomial algorithm for line search in submodular polyhedra. Discrete Optimization 4, 3-4 (Dec. 2007) 349-359.

[25] J. Oxley, 1992: Matroid Theory. Oxford University Press, Oxford.

[26] A. Roth and M. Sotomayor, 1990: Two Sided Matching: A Study in Game-Theoretic Modeling and Analysis. Cambridge University Press, Cambridge.

[27] A. Schrijver, 2003: Combinatorial Optimization--Polyhedra and Efficiency. Springer, Berlin. 
[28] L. J. Schulman and V. V. Vazirani, 2015: Allocation of divisible goods under lexicographic preferences. In Proceedings of 35th IARCS Annual Conf. Foundation of Software Technology and Theoretical Computer Sciences (FSTTCS 2015) (Editors: P. Harsha and G. Ramalingam, Dagstuhl Publishing) 543-559.

[29] L. S. Shapley, 1971: Cores of convex games. International fournal of Game Theory 1, 1 (Dec. 1971) 11-26.

[30] D. J. A. Welsh, 1976: Matroid Theory. Academic Press, London.

[31] L. Zhou, 1990: On a conjecture by Gale about one-sided matching problems. fournal of Economic Theory 52, 1 (Oct. 1990) 123-135.

Received February 2017; revised August 2017; accepted October 2017 